\title{
Quantum density anomaly in optically trapped ultracold gases
}

\author{
Eduardo O. Rizzatti $\odot,{ }^{1, *}$ Marco Aurélio A. Barbosa, ${ }^{2}$ and Marcia C. Barbosa ${ }^{1}$ \\ ${ }^{1}$ Instituto de Física, Universidade Federal do Rio Grande do Sul, Porto Alegre-RS, Brazil \\ ${ }^{2}$ Programa de Pós-Graduacão em Ciência de Materiais, Universidade de Brasília, Planaltina-DF, Brazil
}

(Received 26 April 2019; accepted 20 July 2020; published 22 September 2020)

\begin{abstract}
Water, the substance of life, is known for its myriad of anomalous properties, whose origins are still the subject of intense debates. In order to provide a different insight into this problem, we show how its density anomaly can be reproduced using a quantum simulator. In particular, we demonstrate that the Bose-Hubbard model, a paradigm system in quantum mechanics, exhibits an increase in density with temperature at fixed pressure in the regular fluid regime and in the superfluid phase. We propose that the mechanism underlying the anomalies is related to zero-point entropies and ground-state phase transitions. A connection with the typical experimental scales and setups including confinement effects is also addressed. In this scenario, such finding opens a pathway for theoretical and experimental studies of waterlike anomalies in the area of ultracold quantum gases.
\end{abstract}

DOI: 10.1103/PhysRevA.102.033331

\section{INTRODUCTION}

The experimental realization of the Bose-Einstein condensation $[1,2]$ inaugurated a new era in physics by merging different areas, from condensed matter $[3,4]$ to quantum information [5,6]. This landmark provided grounds for new applications involving the manipulation of ultracold atoms, from which optical lattices, literal crystal arrays of light trapping neutral cold atoms [7,8], stand as a prominent one. Among these applications, systems known as quantum simulators $[9,10]$ have attained great importance since they can be used to experimentally implement and simulate scenarios for a plethora of theoretical ideas [11,12]. Indeed, it is possible to engineer them in highly controllable ways in regards to parameters such as dimensionality, lattice structure, composition, and atomic interactions [13]. In a theoretical level, the Bose-Hubbard model can be considered as a true prototype system, currently used to investigate quantum phase transitions, quantum coherence, and quantum computation [14-16].

In this work we theoretically show that the density of bosons in optical lattices, described by the Bose-Hubbard model, anomalously increases with temperature at fixed pressure in both superfluid and normal fluid regimes. Such counterintuitive behavior, usually denominated as density anomaly, according to our analysis occurs at temperatures below $1.8 \mathrm{nK}$ (superfluid) and $14.8 \mathrm{nK}$ (normal fluid) for rubidium-87 atoms trapped in a simple cubic optical lattice. These anomalies are similar to those presented by liquid water between 0 and $4{ }^{\circ} \mathrm{C}$ at $1 \mathrm{~atm}[17,18]$ and are useful to test the concept that thermodynamic waterlike anomalies arise from the competition between two scales of interaction, associated with critical phenomena. In a quantum-mechanical context such anomaly has also been reported experimentally for liquid helium $[19,20]$. The advantage exhibited by the optical lattice environment is the possibility to control and tune the interactions between particles, enabling a clear analysis regarding possible physical mechanisms.

\footnotetext{
*eduardo.rizzatti@ufrgs.br
}

An explanation for the thermodynamic and dynamic anomalous behavior of liquid water has been disputed through different thermodynamic scenarios. In the second critical point (SCP) hypothesis, which is based on computer simulations of the ST2 atomically detailed model of water [21], followed by extensive investigations on other models for water [22], the apparent divergence of thermodynamic response functions in a metastable region is a consequence of a metastable liquid-liquid phase transition ending in a critical point [21,23]. Nevertheless, this behavior in the case of water was never observed experimentally. The liquid-liquid transitions were reported in models for carbon [24], silicon [25], and silica [26], and experimentally observed in phosphorus [27], triphenyl phosphite, and n-butanol [28]. More recently, experiments with mixtures of water and glycerol [29] and measurements of correlation functions using time-resolved optical Kerr effect (OKE) of supercooled water [30] favor the SCP hypothesis, despite debates in literature [22,31].

The suggested connection between thermodynamic anomalies and criticality in water is difficult to test experimentally since the system freezes before reaching the critical temperature. In addition, the complexity of the water structure makes it difficult to unveil the relation between the microscopic interactions, thermodynamic anomalies, and criticality. Due to its experimental manageability and for being numerically treatable, we propose using the Bose-Hubbard model as a platform to establish this connection. In such context, we explore simple cubic and square geometries, constructed by sets of orthogonal, counterpropagating laser beams of wavelength $\lambda$. The resultant standing waves determine the scenario for the interacting bosons, an optical potential of the form

$$
V_{L}(\mathbf{r})=V_{0} \sum_{i=1}^{d} \sin ^{2}\left(\frac{\pi x_{i}}{a}\right),
$$

where $a=\lambda / 2$ is the lattice spacing, $V_{0}$ is the depth of the optical potential, and $d$ represents the dimensionality of our system with coordination number $z=2 d$. Our theoretical calculations are based on the bosonic self-energy functional 
theory [32], which generates results numerically close to quantum Monte Carlo [33] and bosonic dynamical mean-field theory (BDMFT) techniques.

The paper is organized as follows. First, we introduce the model and its relevant parameters in Sec. II. The theoretical methods employed are explained in Sec. III, with greater details given in the Appendix. The density anomaly in simple cubic and square lattice geometries is discussed in Sec. IV and a mechanism involving residual entropies is proposed in Sec. V for the reported phenomenon. In Sec. VI, we discuss how the anomaly could be experimentally observed based on $2 \mathrm{D}$ in situ imaging of the atoms. Final considerations are addressed in Sec. VII.

\section{BOSE-HUBBARD MODEL}

The dynamics of itinerant bosons in a lattice occupying the lowest-energy band is governed by the Bose-Hubbard Hamiltonian [14,16]

$$
H=-J \sum_{\langle i, j\rangle} b_{i}^{\dagger} b_{j}+\frac{U}{2} \sum_{i} n_{i}\left(n_{i}-1\right)-\mu \sum_{i} n_{i},
$$

where $b_{i}^{\dagger}, b_{i}$, and $n_{i}$ designate the bosonic creation, annihilation, and number operators at site $i$, respectively; $\mu$ is the chemical potential.

The parameter $U$ represents the (typically repulsive) interaction of bosons on the same lattice site. More precisely, it corresponds to the atom-atom $s$-wave scattering process, which can be regarded as an effective contact interaction of the form $U(\mathbf{r})=g \delta(\mathbf{r})$, with a coupling constant $g=4 \pi \hbar^{2} a_{s} / m$ depending on the $s$-wave scattering length $a_{s}$ and mass $m$ of the atoms. By employing the local Wannier states $w(\mathbf{r})$, the term $U$ is expressed through the matrix element

$$
U=g \int d \mathbf{r}|w(\mathbf{r})|^{4} .
$$

On the other hand, the parameter $J$ indicates the hopping amplitude, a kinetic term involving the probability of tunneling between first neighbor sites. Explicitly, it is quantified as the following overlapping integral over adjacent Wannier functions:

$$
J=-\int d \mathbf{r} w\left(\mathbf{r}-\mathbf{r}_{i}\right)\left(-\hbar^{2} \frac{\nabla^{2}}{2 m}+V_{L}(\mathbf{r})\right) w\left(\mathbf{r}-\mathbf{r}_{j}\right) .
$$

Therefore, the Bose-Hubbard model terms $U$ and $J$ can be related to the experimental parameters $\lambda, a_{s}$, and $V_{0}$ through Eqs. (3) and (4) [34], numerically calculating the band structure and obtaining the Wannier states $[35,36]$. These prescriptions allow us to express temperatures in kelvin units and the lattice depth in terms of the recoil energy $E_{r}=\hbar^{2} \pi^{2} / 2 m a^{2}$, according to the values of $U$ and $J$ chosen. It is thus possible to consider our theoretical results within the context of a specific optical trap implementation, from which we select a gas of rubidium-87 atoms in simple cubic $[7,8]$ and square optical lattices $[37,38]$.

\section{METHODOLOGY}

In order to map the thermodynamics of the bosons we employ a variational and nonperturbative self-consistent ap- proach, the self-energy functional theory derived by Hügel et al. [32], inspired in the original works for fermions by Potthoff [39]. The formalism, which includes U(1) symmetry breaking and comprehends previous BDMFT approaches [40-43], is based on successive Legendre transformations of the free-energy functional $\Omega$ leading to a new functional $\Omega_{\mathrm{SE}}$ of the self-energies. The approximation scheme to the manybody problem constricts the variational space: the self-energy domain is restricted to a subspace of self-energies of a simpler reference system. Then, the original problem is transformed into determining stationary solutions of this new functional in terms of the reference system's free propagators. This section is devoted to providing an overview of the method, following Refs. [32,44,45].

First, we write the Hamiltonian of Eq. (2) in a more concise and general form,

$$
H=\frac{1}{2} \mathbf{b}_{\alpha}^{\dagger} \mathbf{t}_{\beta}^{\alpha} \mathbf{b}^{\beta}+\mathcal{V}+\mathbf{F}_{\alpha}^{\dagger} \mathbf{b}^{\alpha},
$$

including an explicit symmetry breaking field $\mathbf{F}$ which couples to the bosonic operators. In this notation, we use the Einstein summation convention and the superindex $\alpha$ spans the site index $i$ as well as the Nambu index $v$. Explicitly, the bosonic operator reads as $\mathbf{b}_{\alpha}^{\dagger} \equiv \mathbf{b}_{i v}^{\dagger}=\left(b_{i}^{\dagger}, b_{i}\right)_{\nu}$, with commutation relations $\left[\mathbf{b}_{\alpha}, \mathbf{b}_{\beta}^{\dagger}\right]=\left(\mathbf{1} \otimes \sigma_{z}\right)_{\beta}^{\alpha}$. Also, we have the generalized hopping $\mathbf{t}_{\beta}^{\alpha}=\mathbf{t}_{j v}^{i \eta}=t_{i j} \otimes \mathbf{1}_{\eta v}$ and interaction of the form $\mathcal{V}=U_{\alpha \beta \gamma \delta} \mathbf{b}^{\alpha} \mathbf{b}^{\beta} \mathbf{b}^{\gamma} \mathbf{b}^{\delta}$.

Including finite-temperature effects (with $k_{B} T=1 / \beta$ ), the partition function $\mathcal{Z}=\operatorname{Tr}\left[\mathcal{T} e^{-\mathcal{S}}\right]$ follows as a trace comprising the imaginary time-ordered exponential of the action $\mathcal{S}$ [46-48],

$$
\begin{aligned}
\mathcal{S}\left[\mathbf{F}, \mathbf{G}_{0}^{-1}\right]= & -\frac{1}{2} \int_{0}^{\beta} \int_{0}^{\beta} d \tau d \tau^{\prime} \mathbf{b}^{\dagger}(\tau) \mathbf{G}_{0}^{-1}\left(\tau, \tau^{\prime}\right) \mathbf{b}\left(\tau^{\prime}\right) \\
& +\int_{0}^{\beta} d \tau \mathcal{V}[\mathbf{b}(\tau)]+\int_{0}^{\beta} d \tau \mathbf{F}^{\dagger} \mathbf{b}(\tau),
\end{aligned}
$$

written according to its explicit dependence on $\mathbf{F}$ and the noninteracting Green's function $\mathbf{G}_{0}$ :

$$
\mathbf{G}_{0}^{-1}=\delta\left(\tau-\tau^{\prime}\right)\left(-\left[\mathbf{1} \otimes \sigma_{z}\right] \partial_{\tau^{\prime}}-\mathbf{t}\right)
$$

From the partition function, averages can be defined as $\langle O(\tau)\rangle=\operatorname{Tr}\left[\mathcal{T} e^{-S} O(\tau)\right] / \mathcal{Z}$. Also, its logarithm provides the free energy $\Omega\left[\mathbf{F}, \mathbf{G}_{0}^{-1}\right]=-\ln [\mathcal{Z}] / \beta$, which is a generating functional of the propagators: the condensate Green's function $\boldsymbol{\Phi}$,

$$
\beta \frac{\delta \Omega}{\delta \mathbf{F}^{\dagger}}=\langle\mathbf{b}\rangle \equiv \boldsymbol{\Phi}
$$

and the connected interacting Green's function $\mathbf{G}$,

$$
2 \beta \frac{\delta \Omega}{\delta \mathbf{G}_{0}^{-1}}=-\left\langle\mathbf{b}(\tau) \mathbf{b}^{\dagger}\left(\tau^{\prime}\right)\right\rangle \equiv \mathbf{G}\left(\tau, \tau^{\prime}\right)-\boldsymbol{\Phi} \boldsymbol{\Phi}^{\dagger} .
$$

Further details regarding products and traces are addressed in Appendixes A and B.

Based on a Legendre transformation, the free-energy functional dependence can be exchanged from $\mathbf{F}$ and $\mathbf{G}_{0}^{-1}$ to the dressed propagators $\boldsymbol{\Phi}$ and $\mathbf{G}$, leading to the Baym-Kadanoff 
functional [49-52]

$$
\begin{aligned}
\beta \Omega_{\mathrm{BK}}[\boldsymbol{\Phi}, \mathbf{G}]= & \mathbf{F}^{\dagger} \boldsymbol{\Phi}-\frac{1}{2} \boldsymbol{\Phi}^{\dagger} \mathbf{G}_{0}^{-1} \boldsymbol{\Phi}+\frac{1}{2} \operatorname{Tr}\left[\mathbf{G}_{0}^{-1} \mathbf{G}\right] \\
& +\frac{1}{2} \operatorname{Tr} \ln \left[-\mathbf{G}^{-1}\right]+\Phi_{\mathrm{LW}}[\boldsymbol{\Phi}, \mathbf{G}] .
\end{aligned}
$$

In Eq. (10), $\Phi_{\mathrm{LW}}[\boldsymbol{\Phi}, \mathbf{G}]$ is the Luttinger-Ward functional $[53,54]$, a universal contribution which encompasses the complexity of the many-body system, containing all two-particle irreducible (2PI) diagrams [55,56]. At the physical solution, the functional $\Omega_{\mathrm{BK}}[\boldsymbol{\Phi}, \mathbf{G}]$ is stationary,

$$
\frac{\delta \Omega_{\mathrm{BK}}}{\delta \boldsymbol{\Phi}^{\dagger}}=0, \quad \frac{\delta \Omega_{\mathrm{BK}}}{\delta \mathbf{G}}=0,
$$

and it is equal to the equilibrium free energy $\Omega_{\mathrm{BK}}=\Omega$. The variations,

$$
\beta \frac{\delta \Omega_{\mathrm{BK}}}{\delta \boldsymbol{\Phi}^{\dagger}}=\mathbf{F}-\mathbf{G}_{0}^{-1} \boldsymbol{\Phi}+\frac{\delta \Phi_{\mathrm{LW}}}{\delta \boldsymbol{\Phi}^{\dagger}}
$$

and

$$
2 \beta \frac{\delta \Omega_{\mathrm{BK}}}{\delta \mathbf{G}}=\mathbf{G}_{0}^{-1}-\mathbf{G}^{-1}+2 \frac{\delta \Phi_{\mathrm{LW}}}{\delta \mathbf{G}},
$$

associated to the conditions of Eq. (11) lead to the following identification:

$$
\boldsymbol{\Sigma}_{1 / 2}=-\frac{\delta \Phi_{\mathrm{LW}}}{\delta \boldsymbol{\Phi}^{\dagger}}, \quad \boldsymbol{\Sigma}=-2 \frac{\delta \Phi_{\mathrm{LW}}}{\delta \mathbf{G}},
$$

where $\boldsymbol{\Sigma}_{1 / 2}$ and $\boldsymbol{\Sigma}$ are the one- and two-point self-energies, respectively. Therefore, the propagators obey the Dyson equations

$$
\mathbf{G}_{0}^{-1} \boldsymbol{\Phi}=\mathbf{F}-\boldsymbol{\Sigma}_{1 / 2}
$$

and

$$
\mathbf{G}^{-1}=\mathbf{G}_{0}^{-1}-\mathbf{\Sigma} .
$$

With another Legendre transform, the Baym-Kadanoff functional dependence can be exchanged from the one- and twopoint propagators $\boldsymbol{\Phi}$ and $\mathbf{G}$ to their respective self-energies $\Sigma_{1 / 2}$ and $\boldsymbol{\Sigma}$, yielding the self-energy functional

$$
\begin{aligned}
\beta \Omega_{\mathrm{SE}}\left[\boldsymbol{\Sigma}_{1 / 2}, \boldsymbol{\Sigma}\right]= & \frac{1}{2}\left(\mathbf{F}-\boldsymbol{\Sigma}_{1 / 2}\right)^{\dagger} \mathbf{G}_{\mathbf{0}}\left(\mathbf{F}-\boldsymbol{\Sigma}_{1 / 2}\right) \\
& +\frac{1}{2} \operatorname{Tr} \ln \left[-\left(\mathbf{G}_{\mathbf{0}}{ }^{-1}-\boldsymbol{\Sigma}\right)\right] \\
& +\mathcal{F}\left[\boldsymbol{\Sigma}_{1 / 2}, \boldsymbol{\Sigma}\right] .
\end{aligned}
$$

The universal functional $\mathcal{F}\left[\boldsymbol{\Sigma}_{1 / 2}, \boldsymbol{\Sigma}\right]=\Phi_{\mathrm{LW}}[\boldsymbol{\Phi}, \mathbf{G}]+$ $\boldsymbol{\Sigma}_{1 / 2}^{\dagger} \boldsymbol{\Phi}+\frac{1}{2} \operatorname{Tr}[\boldsymbol{\Sigma} \mathbf{G}]$ is simply the Legendre transform of the Luttinger-Ward functional $\Phi_{\mathrm{LW}}[\boldsymbol{\Phi}, \mathbf{G}]$, with the following variations:

$$
\frac{\delta \mathcal{F}}{\delta \boldsymbol{\Sigma}_{1 / 2}^{\dagger}}=\boldsymbol{\Phi}, \quad 2 \frac{\delta \mathcal{F}}{\delta \boldsymbol{\Sigma}}=\mathbf{G} .
$$

At the physical solution, $\Omega_{\mathrm{SE}}$ is stationary and equal to the free energy $\Omega_{\mathrm{SE}}=\Omega_{\mathrm{BK}}=\Omega$ (as a result of $\Omega, \Omega_{\mathrm{BK}}$, and $\Omega_{\mathrm{SE}}$ being connected by successive Legendre transforms), yielding once again the Dyson equations

$$
0=\beta \frac{\delta \Omega_{\mathrm{SE}}}{\delta \boldsymbol{\Sigma}_{1 / 2}^{\dagger}}=-\mathbf{G}_{\mathbf{0}}\left(\mathbf{F}-\boldsymbol{\Sigma}_{1 / 2}\right)+\boldsymbol{\Phi}
$$

and

$$
0=2 \beta \frac{\Omega_{\mathrm{SE}}}{\delta \boldsymbol{\Sigma}}=-\left(\mathbf{G}_{\mathbf{0}}^{-1}-\boldsymbol{\Sigma}\right)^{-1}+\mathbf{G}
$$

The mentioned universality of the functional $\mathcal{F}$ enables us to overcome its complexity with the introduction of an exactly solvable reference system (denoted by primed quantities) exhibiting the same symmetry and interactions as the original one. According to Eq. (17), the reference system's self-energy functional

$$
\begin{aligned}
\beta \Omega_{\mathrm{SE}}^{\prime}\left[\boldsymbol{\Sigma}_{1 / 2}, \boldsymbol{\Sigma}\right]= & \frac{1}{2}\left(\mathbf{F}^{\prime}-\boldsymbol{\Sigma}_{1 / 2}\right)^{\dagger} \mathbf{G}_{0}^{\prime}\left(\mathbf{F}^{\prime}-\boldsymbol{\Sigma}_{1 / 2}\right) \\
& +\frac{1}{2} \operatorname{Tr} \ln \left[-\left(\mathbf{G}_{0}^{\prime-1}-\mathbf{\Sigma}\right)\right]+\mathcal{F}\left[\boldsymbol{\Sigma}_{1 / 2}, \mathbf{\Sigma}\right]
\end{aligned}
$$

evaluated at the physical solutions $\boldsymbol{\Sigma}_{1 / 2}=\boldsymbol{\Sigma}_{1 / 2}^{\prime}$ and $\boldsymbol{\Sigma}=\boldsymbol{\Sigma}^{\prime}$ is equal to the reference system's free energy $\Omega_{\mathrm{SE}}^{\prime}\left[\Sigma_{1 / 2}^{\prime}, \Sigma^{\prime}\right]=$ $\Omega^{\prime}\left[\mathbf{F}^{\prime}, \mathbf{G}_{0}^{\prime-1}\right]$. Subtracting Eq. (21) from Eq. (17), $\Omega_{\mathrm{SE}}$ evaluated at $\boldsymbol{\Sigma}_{1 / 2}=\boldsymbol{\Sigma}_{1 / 2}^{\prime}$ and $\boldsymbol{\Sigma}=\boldsymbol{\Sigma}^{\prime}$ becomes

$$
\begin{aligned}
\beta \Omega_{\mathrm{SE}}\left[\boldsymbol{\Sigma}_{1 / 2}^{\prime}, \mathbf{\Sigma}^{\prime}\right]= & \beta \Omega^{\prime}+\frac{1}{2}\left(\mathbf{F}-\boldsymbol{\Sigma}_{1 / 2}^{\prime}\right)^{\dagger} \mathbf{G}_{0}\left(\mathbf{F}-\boldsymbol{\Sigma}_{1 / 2}^{\prime}\right) \\
& -\frac{1}{2}\left(\mathbf{F}^{\prime}-\boldsymbol{\Sigma}_{1 / 2}^{\prime}\right)^{\dagger} \mathbf{G}_{0}^{\prime}\left(\mathbf{F}^{\prime}-\boldsymbol{\Sigma}_{1 / 2}^{\prime}\right) \\
& +\frac{1}{2} \operatorname{Tr} \ln \left[\frac{\mathbf{G}_{0}^{-1}-\boldsymbol{\Sigma}^{\prime}}{\mathbf{G}_{0}^{\prime-1}-\boldsymbol{\Sigma}^{\prime}}\right]
\end{aligned}
$$

Therefore, the solution of the reference system provides a parametrization of the self-energies in terms of $\mathbf{F}^{\prime}$ and $\mathbf{G}_{0}^{\prime-1}$, which allows for the construction of the self-energy functional theory approximation $\Omega_{\mathrm{SFT}}$ to the self-energy functional $\Omega_{\mathrm{SE}}$ according to

$$
\Omega_{\mathrm{SFT}}\left[\mathbf{F}^{\prime}, \mathbf{G}_{0}^{\prime-1}\right]=\Omega_{\mathrm{SE}}\left[\boldsymbol{\Sigma}_{1 / 2}^{\prime}\left[\mathbf{F}^{\prime}, \mathbf{G}_{0}^{\prime-1}\right], \boldsymbol{\Sigma}^{\prime}\left[\mathbf{F}^{\prime}, \mathbf{G}_{0}^{\prime-1}\right]\right] .
$$

The approximation consists in constraining the variational principle to the subspace of self-energies of the reference system; this procedure applied to the variations of Eqs. (19) and (20) yields the Euler equations $\delta_{\mathbf{F}^{\prime \dagger}} \Omega_{\mathrm{SFT}}=0$ and $\delta_{\mathbf{G}_{0}^{\prime-1}} \Omega_{\mathrm{SFT}}=$ 0 .

In particular, we choose a local reference system, the SFA3 minimal construction [32], comprehending three variational parameters: the U(1) symmetry breaking linear field $F^{\prime}$ conjugated to the creation $b^{\dagger}$ and annihilation $b$ operators, the two fields $\Delta_{00}$, coupled with the density $b^{\dagger} b$, and $\Delta_{01}$, conjugated to pair creation $b^{\dagger} b^{\dagger}$ and pair annihilation $b b$ operators. The Hamiltonian describing the bosonic state is given by

$$
H^{\prime}\left[\mathbf{F}^{\prime}, \mathbf{\Delta}\right]=\frac{1}{2} \mathbf{b}^{\dagger} \mathbf{\Delta} \mathbf{b}+\frac{U}{2} n(n-1)-\mu n+\mathbf{F}^{\prime \dagger} \mathbf{b},
$$

where $\mathbf{b}=\left(b, b^{\dagger}\right), \mathbf{F}^{\prime}=\left(F^{\prime}, F^{* *}\right)$, and $\boldsymbol{\Delta}=\Delta_{00} \mathbf{1}+\Delta_{01} \sigma_{x}$.

Therefore, the states of thermodynamic equilibrium are determined by the stationary points of $\Omega_{\mathrm{SFT}}$, given by $\nabla \Omega_{\mathrm{SFT}}\left[F, \Delta_{00}, \Delta_{01}\right]=0\left(\right.$ or $\delta_{\mathbf{F}^{\prime /}} \Omega_{\mathrm{SFT}}=0$ and $\left.\delta_{\Delta} \Omega_{\mathrm{SFT}}=0\right)$. The functional can be evaluated according to the following steps, which are completely developed throughout the Appendix sections. Given the parameters $F^{\prime}, \Delta_{00}$, and $\Delta_{01}$, the Hamiltonian of Eq. (24) is determined. From Appendix C, the reference system's partition function and free energy are computed through Eqs. (C1) and (C2), followed by its oneand two-point propagators of Eqs. (C3) and (C5), and the self-energies according to the Dyson Eqs. (C7) and (C8). The next step is to calculate the lattice system's one- and two-point propagators by using Eqs. (D4) and (D3), respectively. These 

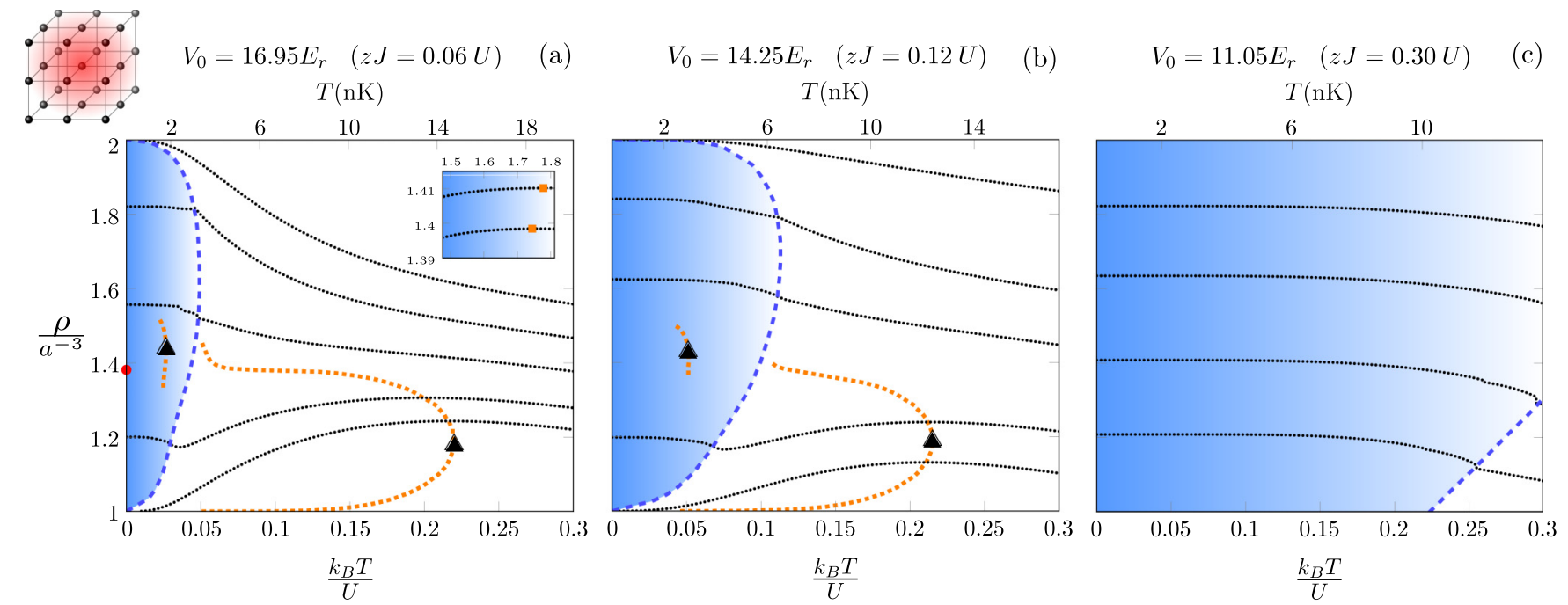

FIG. 1. Density $\rho$ as a function of the reduced temperature $k_{B} T / U$ (bottom) and the temperature $T$ in nanokelvin units (top) at fixed pressures for hopping amplitudes: (a) $z J=0.06 U$, (b) $z J=0.12 U$, and (c) $z J=0.30 U$, considering a simple cubic lattice ( $z=6$ ). The superfluid phase is highlighted in blue, while the normal phase is portrayed in white. Blue dashed lines denote the boundaries between superfluid and normal phases, while orange dotted lines represent the TMD curves. In (a), the inset exhibits a zoom of isobaric curves in the superfluid phase, while the red point signals the atomic limit critical point at $T=0$. The triangular points denote the maximum temperatures reached by each TMD curve.

products and the logarithmic trace $\operatorname{Tr} \ln \left[\mathbf{G}^{\prime} \mathbf{G}^{-1}\right]$ are determined following the prescriptions presented in Appendixes $\mathrm{A}$ and $\mathrm{B}$, respectively. By collecting the required terms in Eq. (22), the desired self-energy functional is evaluated and its stationary points can be determined. The code employed in our calculations is available in the Supplemental Material [57].

\section{DENSITY ANOMALY}

From the equilibrium free energy $\Omega=\Omega_{\mathrm{SFT}}$ calculated previously, the density is given by

$$
\rho=-\frac{1}{V}\left(\frac{\partial \Omega}{\partial \mu}\right)_{T}
$$

where $V=N_{s} a^{d}$ is the volume and $N_{s}$ is the number of lattice sites. Its temperature dependence at fixed pressure $P$ is determined by the isobaric thermal expansion coefficient

$$
\alpha=-\frac{1}{\rho}\left(\frac{\partial \rho}{\partial T}\right)_{P}
$$

For $\alpha<0$, density increases with temperature and a region of anomalous density behavior is identified by a temperature of maximum density (TMD) line defined as $\alpha=0$. The pressure is fixed employing the Gibbs-Duhem relation $d P=\rho d \mu+$ $s d T=0$, where $s=-\frac{1}{V}\left(\frac{\partial \Omega}{\partial T}\right)_{\mu}$ is the entropy density and $P$ is related to the grand-canonical potential according to $-P V=$ $\Omega=\Omega_{\mathrm{SFT}}$.
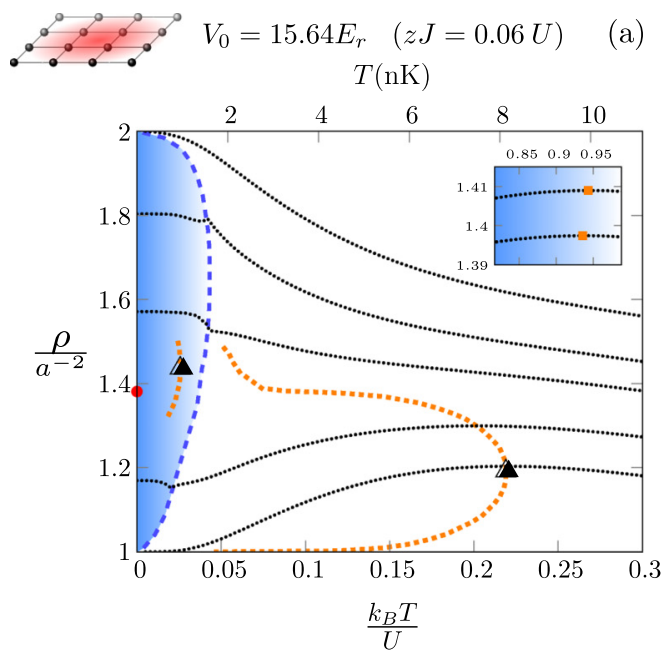

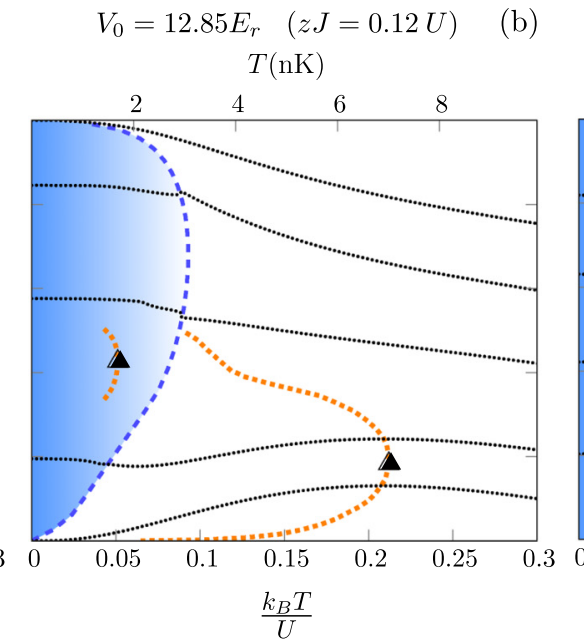

(b) $T(\mathrm{nK})$

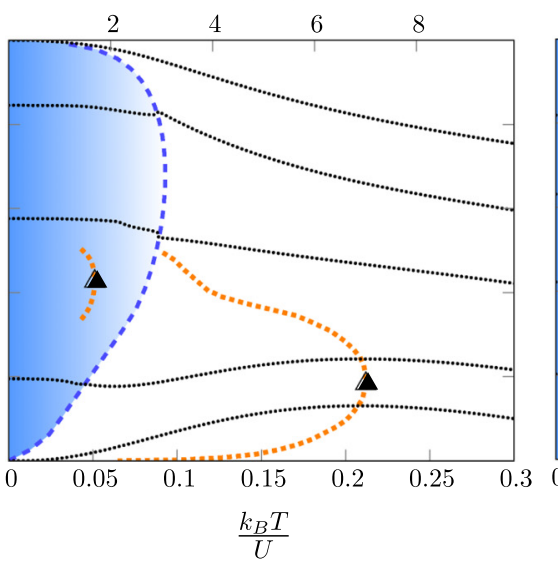

$$
\begin{gathered}
V_{0}=9.56 E_{r} \quad(z J=0.30 U) \\
T(\mathrm{nK})
\end{gathered}
$$
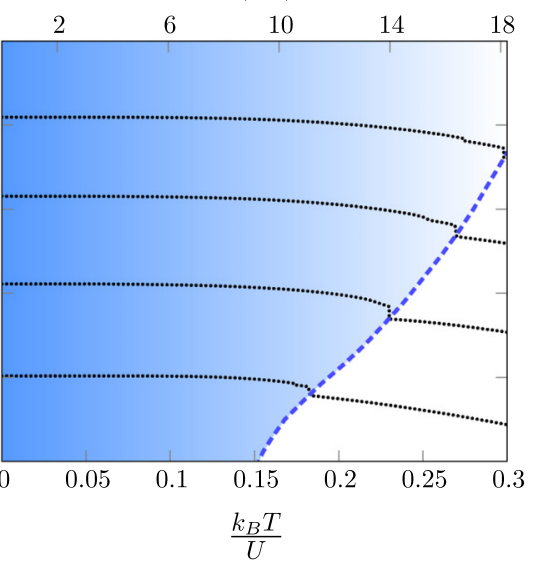

FIG. 2. Density $\rho$ as a function of the reduced temperature $k_{B} T / U$ (bottom) and the temperature $T$ in nanokelvin units (top) at fixed pressures for hopping amplitudes: (a) $z J=0.06 U$, (b) $z J=0.12 U$, and (c) $z J=0.30 U$, considering a square lattice $(z=4)$. 
TABLE I. Experimental parameters regarding potential depths $V_{0}$, scattering length $a_{s}$, and laser wavelengths $\lambda$ of optical lattices implemented using different alkali-metal elements, for the hopping amplitudes $z J=0.06 U$ and $z J=0.12 U$. The maximum temperatures in which density anomalies are observed in superfluid $T_{S F}$ and normal phases $T_{\mathrm{NA}}$ (the highlighted triangular points in Fig. 1 and Fig. 2) are also addressed. In the 2D scenarios, the vertical confinement is achieved by an optical potential along the $z$ axis. For rubidium-87 [38] the lattice depth is $V_{0, z}=26 E_{r}$, while the cesium-133 [58] atoms are confined in a Gaussian wave packet of width $a_{z}=0.30 \mu \mathrm{m}$.

\begin{tabular}{|c|c|c|c|c|c|c|c|c|c|}
\hline \multirow[b]{2}{*}{ Element } & \multirow[b]{2}{*}{ Geometry } & \multirow[b]{2}{*}{$\lambda(\mathrm{nm})$} & \multirow[b]{2}{*}{$a_{s}\left(a_{0}\right)$} & \multicolumn{3}{|c|}{$z J=0.06 U$} & \multicolumn{3}{|c|}{$z J=0.12 U$} \\
\hline & & & & $V_{0} / E_{r}$ & $T_{\mathrm{NA}}(\mathrm{nK})$ & $T_{\mathrm{SA}}(\mathrm{nK})$ & $V_{0} / E_{r}$ & $T_{\mathrm{NA}}(\mathrm{nK})$ & $T_{\mathrm{SA}}(\mathrm{nK})$ \\
\hline${ }^{23} \mathrm{Na}[59]$ & Simple cubic $(z=6)$ & 595 & 52 & 18.30 & 88.35 & 10.68 & 15.49 & 74.67 & 17.82 \\
\hline${ }^{87} \mathrm{Rb}$ [7] & Simple cubic $(z=6)$ & 852 & 103 & 16.95 & 14.76 & 1.78 & 14.25 & 12.39 & 2.95 \\
\hline${ }^{87} \mathrm{Rb}[38]$ & Square $(z=4)$ & 1064 & 103 & 15.64 & 8.13 & 0.95 & 12.85 & 7.00 & 1.69 \\
\hline${ }^{133} \mathrm{Cs}[58]$ & Square $(z=4)$ & 1064 & 310 & 16.65 & 4.36 & 0.51 & 13.77 & 3.77 & 0.91 \\
\hline
\end{tabular}

Considering a simple cubic lattice, with $z=6$, Figs. 1(a)1(c) illustrate the density $\rho$ versus the reduced temperature $k_{B} T / U$ at fixed pressures (represented as black lines) for increasing hopping amplitudes: (a) $z J=0.06 U$, (b) $z J=$ $0.12 U$, and (c) $z J=0.30 U$. The corresponding potential depths, which decrease from (a) to (c), and temperature scales in nanokelvin units are calculated considering atoms of rubidium-87, with realistic values based on the experiments performed by Greiner et al. [7]. The superfluid to normal phase boundary is illustrated as a reentrant dashed blue line and the blue filled area represents the superfluid phase. Figures 1(a) and 1(b) show that at sufficient high values of $V_{0}$ (low values of $z J / U$ ) there are two regions in which density presents a local maximum, with the TMD curves represented as orange dots: one at the normal phase [normal phase anomaly (NA)] and another at the superfluid phase [superfluid phase anomaly (SA)]. The maximum temperature values reached by the TMD curves are highlighted as triangular black points.

Figure 1(a) portrays a large area in the density versus temperature phase diagrams where the NA is present. However, as the hopping increases, according to Fig. 1(b), the anomaly occupies a smaller region in temperatures. In addition to the normal phase TMD, the superfluid phase also exhibits a density anomalous behavior illustrated in Fig. 1(a), with a few superfluid isobaric densities drawn in the inset. When the hopping becomes larger it dominates the free energy, leading the superfluid to occupy a bigger region in the phase diagram and suppressing both superfluid and normal anomalies, as presented in Fig. 1(c).

Analogously, Fig. 2 displays the two-dimensional results considering a square lattice geometry, with $z=4$. For comparison reasons, we chose the same ratios $z J / U$ as shown in Fig. 1. The superfluid domain exhibits a small retraction when compared to the simple cubic case. In spite of this, the same general behavior is observed. Specifically, Fig. 2(a) presents the density anomaly in both phases; the anomaly is reduced for a larger hopping amplitude as illustrated in Fig. 2(b) and finally it vanishes completely as shown in Fig. 2(c). For this two-dimensional system, the respective potential depths and temperature scales in nanokelvin units are calculated also considering atoms of rubidium-87, with parameters according to the experiments performed by Sherson et al. [38].

Although the upper temperature scales and potential depths addressed in Figs. 1 and 2 refer to specific setups using rubidium-87, they can be adapted to other elements. Indeed, we collect in Table I the temperatures, $T_{\mathrm{SA}}$ and $T_{\mathrm{NA}}$, that must be achieved for experimentally detecting SA and NA not only for the previous cases of rubidium-87 but also for sodium-23 [59] (in a simple cubic lattice) and cesium-133 [58,60,61] (in a square lattice). These points are marked as the triangular symbols over the TMD curves.

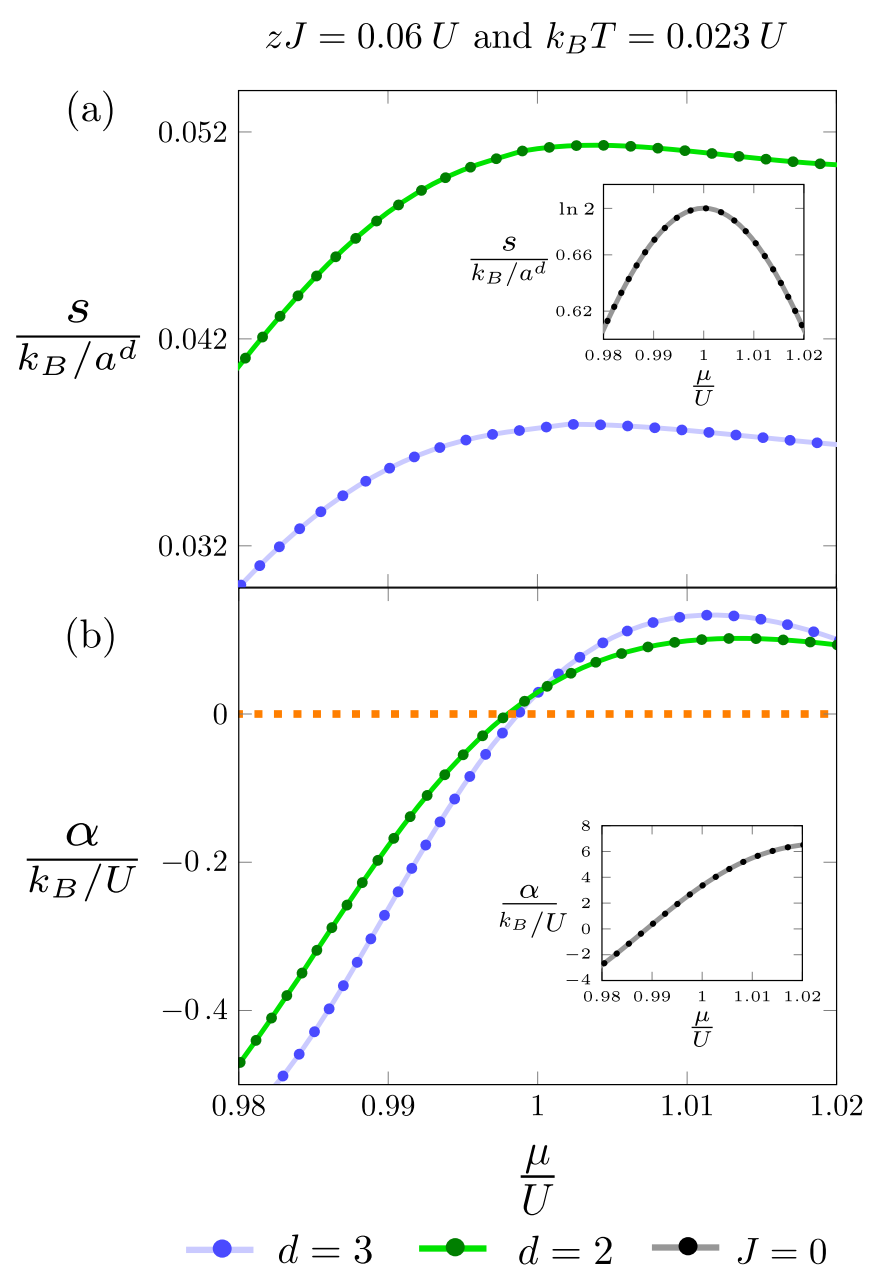

FIG. 3. Considering square $(d=2)$ and simple cubic $(d=3)$ lattices, the entropy (a) and thermal expansion coefficient (b) are exhibited as functions of the chemical potential $\mu$ for $z J=0.06 U$ at $k_{B} T=0.023 U$ deep in the superfluid regime. The respective insets depict the atomic limit $(J=0)$ scenario. 
(a)

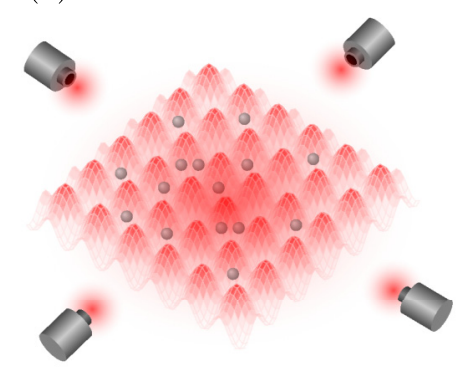

(b)

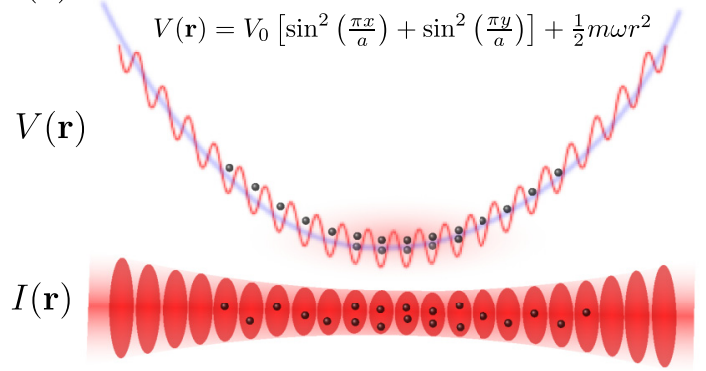

(c) $V_{0}=15.64 E_{r} \quad(z J=0.06 U)$

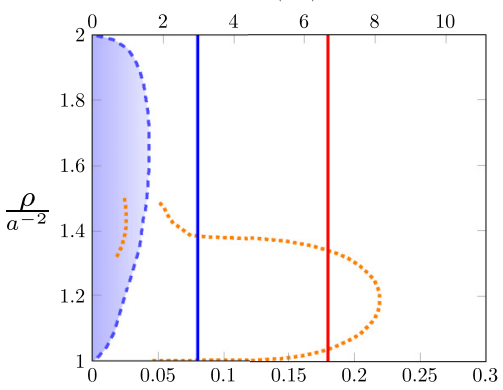

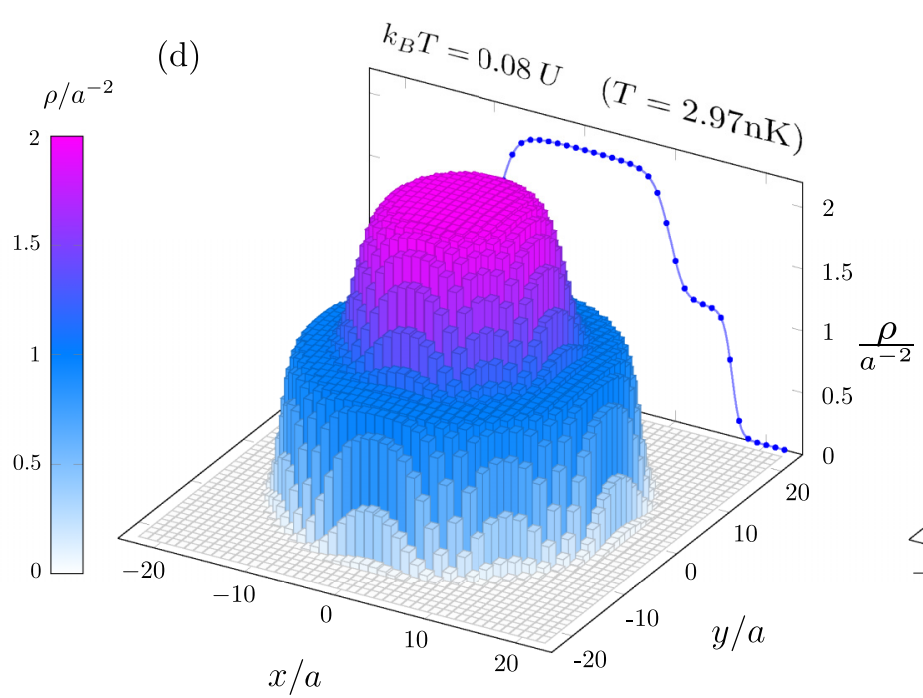

(e)

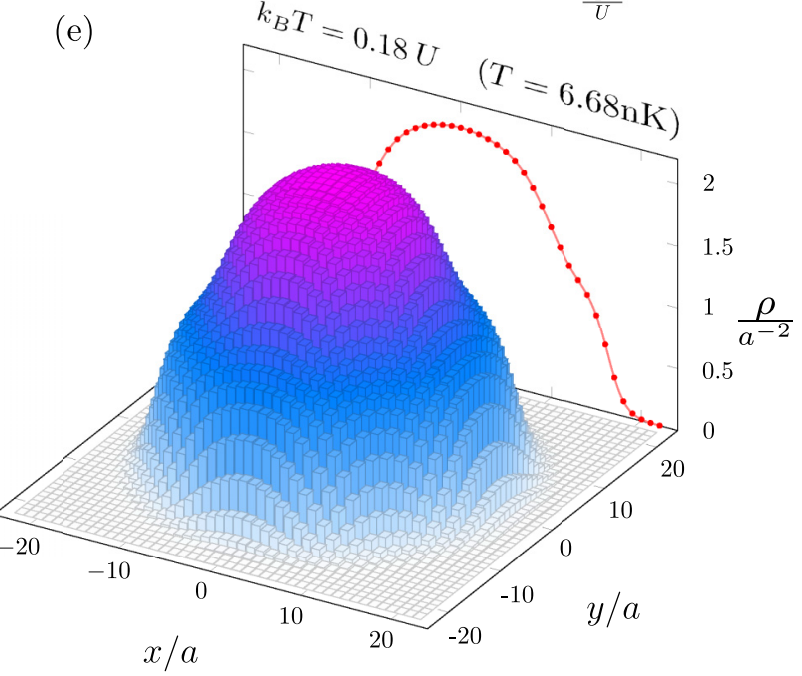

FIG. 4. Density distribution in the normal phase, considering harmonic confinement effects. (a) The two-dimensional configuration of a square optical lattice is created by counterpropagating red detuned laser beams. The additional harmonic effects are represented in (b), where the optical potential $V(\mathbf{r})$ and the intensity profile $I(\mathbf{r})$ are schematically shown. (c) The phase diagram $\rho$ versus $T$ for the configuration $V_{0}=15.64 E_{r}(z J=0.06 U)$ highlights two chosen temperatures $k_{B} T=0.08 U$ (blue) and $k_{B} T=0.18 U$ (red). Considering the LDA scenario, the density $\rho$ is mapped in the $x y$ plane for the respective temperatures in (d) and (e).

\section{RESIDUAL ENTROPY MECHANISM}

The density anomalies in the normal fluid can be traced back to the ground-state phase transitions between Mott insulators of successive occupation numbers [62]. This anomalous behavior, present even in the absence of hopping, arises from the competition between the chemical potential, which promotes the boson occupation in the lattice, with the on-site repulsion interaction $U$, which favors the boson removal. As the temperature increases, entropy first favors filling up the sites but, for high enough temperatures, entropy increases by removing particles from the system to increase the mobility of the particles left. This is a classical behavior similar to that of liquid water, where bonding and nonbonding structures compete: at lower temperatures density increases by disrupting hydrogen bonds, while at higher temperatures enhanced particles' velocities increase the available volume, decreasing density. The difference here is that this phase is not completely destroyed by the hopping, persisting for values of the $J$ possible to be observed experimentally.

Indeed, the hopping brings another phenomenon not observed for $J=0$ : the SA, a quantum density anomaly. The physical origin of this behavior is also the competition between chemical potential and the repulsion $U$. But for the
SA the TMD line appears at lower temperatures and higher densities when compared with the NA, because in this case the hopping contributes to the temperature effects, favoring the movement and the spread of particle over the lattice.

Such competition of interaction scales can also be translated in terms of degeneracies and residual entropies. Inhibiting the hopping, a ground-state degeneracy, related to a phase transition in number occupation between Mott insulators, is settled whenever the chemical potential $\mu$ reaches an integer value of the interaction $U$. At such transition points, two states are equally accessible and this degeneracy accounts for an observed macroscopic residual entropy of $k_{B} \ln 2$ per site. For finite temperatures, these entropies develop into peaks near those points as the chemical potential is varied; see the inset of Fig. 3(a). By turning on the tunneling probability adiabatically the superfluid phase emerges exactly from Mott insulator transition points, mitigating residual entropies, since the previous degeneracy gets lifted. Thus, for a finite hopping transition, the mentioned entropy maxima remain deep in the superfluid phase but are less prominent, as shown in Fig. 3(a) for $z J=0.06 U$ and $k_{B} T=0.023 U$. Formally, the entropy peaks mark a change in the behavior of density with temperature according to the Maxwell 
relation

$$
\left(\frac{\partial s}{\partial \mu}\right)_{T}=\left(\frac{\partial \rho}{\partial T}\right)_{\mu}=-\rho \alpha_{\mu},
$$

which results in the sign flip of thermal expansion in the superfluid phase illustrated in Fig. 3(b).

\section{2D IN SITU OBSERVATION INCLUDING CONFINEMENT EFFECTS}

On the basis of the recently developed in situ measurements of ultracold gases in optical lattices [63], we discuss a physical realization of the reported phenomenon considering rubidium87 atoms disposed in a square lattice, as Fig. 4(a) depicts. These modern tools encompass high resolution absorption [58,61], fluorescence imaging [37,38], and even scanning electron microscopy [64], each technique with its specific applications. Considering the range of density and fluctuations presented here, our theoretical proposal appears better suited to the absorption imaging realized by Chin et al. $[58,61]$, complementing the well-known time-of-flight methods which probe the system in momentum space. In such experimental framework, in situ density distributions $\rho(x, y)$ of 2D gases can be determined by performing absorption imaging perpendicular to the horizontal plane $x y$. This technique allows for mapping the occupation number at a single site resolution, providing direct access to density fluctuations, which is our ultimate goal in the analysis of the anomaly.

In our discussion, the confinement effects of the harmonic trapping field, represented in Fig. 4(b), are considered using a local density approximation (LDA). The harmonic confinement potential is given by $V_{h}(r)=\frac{1}{2} m \omega^{2} r^{2}$, where $r=$ $\sqrt{x^{2}+y^{2}}$ is the radial distance from the center of the trap and the associated oscillation frequency $\omega$ is fixed at $\omega / 2 \pi=$ $60 \mathrm{~Hz}$, as typically chosen. Consequently, in the LDA framework the chemical potential across the lattice takes the form $\mu(r)=\mu_{0}-V_{h}(r)$. The lattice depth is held at $V_{0}=15.64 E_{r}$ $(z J=0.06 U)$, as already discussed in Fig. 2(a). Furthermore, the total number of particles is kept constant, $N_{\text {total }}=1096$, as well as the total pressure, $P_{\text {total }}=1.9765 U / a^{2}$. Under these described conditions, such quantities satisfy the equation of state $[65,66]$

$$
P_{\text {total }}=\frac{m \omega^{2}}{2 \pi} N_{\text {total }}
$$

as demonstrated in Appendix F. This simple relation implies that, if $N_{\text {total }}$ is fixed, then $P_{\text {total }}$ is naturally held constant. Given all these parameters, we restrict our analysis to the normal phase anomaly since the variations in density with temperature are more prominent.

Since we want to investigate how the density behaves when temperature is changed at fixed pressure, Figs. 4(d) and 4(e) exhibit the density $\rho$ on each lattice site of the $x y$ plane for temperatures $k_{B} T=0.08 U(T=2.97 \mathrm{nK})$ and $k_{B} T=0.18 U$ $(T=6.68 \mathrm{nK})$, respectively. These two temperatures are also marked as blue and red straight lines in the phase diagram of Fig. 4(c), showing that only the normal phase is present. In
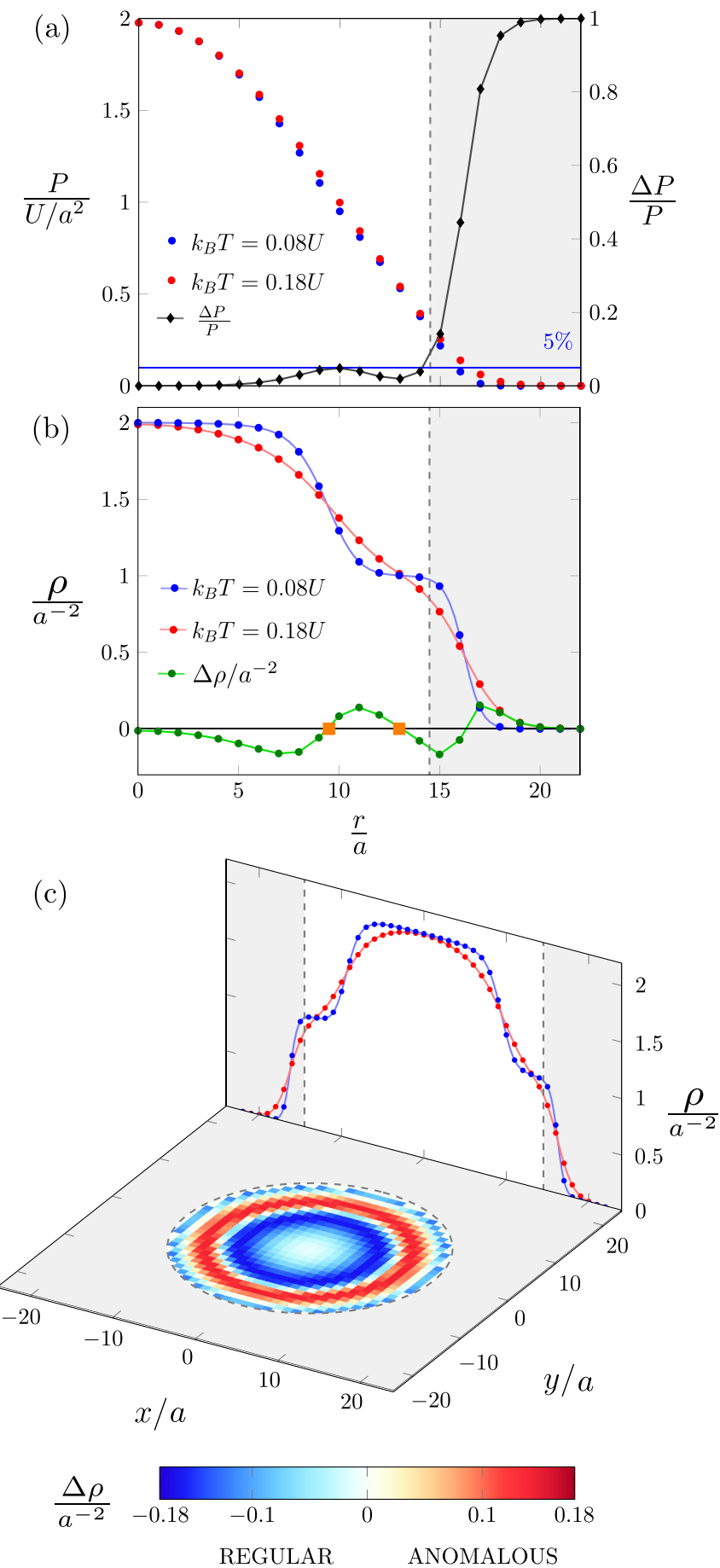

FIG. 5. Analysis of the setup presented in Fig. 4. Panel (a) shows the pressure $P$ for two different temperatures $\left(k_{B} T=0.08 U\right.$ and $\left.k_{B} T=0.18 U\right)$ and their relative differences $\Delta P / P$ as a function of the radial distance $r$. The gray area signals the distances where such pressure deviation becomes greater than $5 \%$. (b) The density profiles for these temperatures are exhibited as functions of the distance $r$ as well as their difference $\Delta \rho$, with the anomalous region $(9 a<$ $r<14 a$ ) delimited by two TMD points (orange squares). (c) $\Delta \rho$ is represented as a color map in the $x y$ plane.

a qualitative perspective, we observed a melting of the steps as temperature is raised and a larger number fluctuations between the steps of integer density. Quantitatively, a closer look 
at how the local pressure $P$ varies across the radial direction is discussed in Fig. 5(a). Due to the finite deviation between such temperatures $k_{B} \Delta T=0.1 U$, there are differences in pressure $\Delta P$ compared to their respective values at each site which are less than 5\% until $r=14 a$. Larger distances are filled in gray, comprehending an area where the relative variation $\Delta P / P$ grows towards the boundaries of the lattice. Hence the pressure is kept approximately constant at each site except inside the gray area, where $r>14 a$. Figure 5(b) shows the radial density profiles in greater detail, with their difference $\Delta \rho$ in green. This curve shows oscillations, taking on positive values. These positive values indicate the density anomaly and are limited by two TMD points, shown as orange square points. Finally, Fig. 5(c) summarizes our analysis where the difference in density $\Delta \rho$ is represented through a color map in the $x y$ plane. The anomalous region $\Delta \rho>0$ denotes an increase in density with temperature at fixed pressure, while the regular behavior corresponds to $\Delta \rho<0$. As previously, the gray area excludes the regions where the local pressure does not remain fixed. Under these circumstances, we have shown a signature of the density anomaly in this $2 \mathrm{D}$ configuration, illustrated as the red circular ring of Fig. 5(c).

\section{CONCLUSION}

We have predicted theoretically the occurrence of density anomaly in a quantum system considering parameters compatible with its experimental realization in optical lattices, within the framework described by the self-energy functional theory. It was also shown that the physical mechanism underlying normal density anomalies relies on the presence of a zero-point entropy in the atomic limit, marking phase transitions between Mott insulators with different occupation. The inclusion of the hopping amplitude (enabling the rise of a superfluid phase) lifts the ground-state degeneracy, generates correlations among different sites, and damps residual entropies and thermal expansion. Nevertheless, regions of anomalous density behavior can be found in a perturbative regime $(J \ll U)$ corresponding to atomic recoil energy being much smaller than the intensities of the confining field $E_{r} \ll$ $V_{0}$. For very intense confining fields waterlike anomalies are also found inside the superfluid regime, as was illustrated for the case of rubidium-87 in Figs. 1 and 2. Our proposition is that by understanding the competition between different physical mechanisms contributing to free energy, usually manifested through interactions between particles (but here including chemical potential and hopping), and the relation between residual entropy and ground-state phase transitions, it is possible to design and predict the phenomenology of density anomaly in systems other than liquid water, as illustrated here with optical lattices of rubidium-87, sodium-23, and cesium-133 atoms.

\section{ACKNOWLEDGMENTS}

We acknowledge the financial support of the Brazilian funding agencies FAPDF, CNPq, and CAPES.

\section{APPENDIX A: TENSOR PRODUCTS}

The product $\mathbf{V}^{\alpha}(\tau)$ of a second-order tensor $\mathbf{M}_{\beta}^{\alpha}\left(\tau, \tau^{\prime}\right)$ and a first-order tensor $\mathbf{F}^{\alpha}(\tau)$ comprises the sum over the superindex and integration in imaginary time according to

$$
\mathbf{V}^{\alpha}(\tau)=\sum_{\gamma} \int_{0}^{\beta} d \tilde{\tau} \mathbf{M}_{\gamma}^{\alpha}(\tau, \tilde{\tau}) \mathbf{F}^{\gamma}(\tilde{\tau}) .
$$

Similarly, the product $\mathbf{M}_{\beta}^{\alpha}\left(\tau, \tau^{\prime}\right)$ of two second-order tensors $\mathbf{A}_{\beta}^{\alpha}\left(\tau, \tau^{\prime}\right)$ and $\mathbf{B}_{\beta}^{\alpha}\left(\tau, \tau^{\prime}\right)$ is defined by

$$
\mathbf{M}_{\beta}^{\alpha}\left(\tau, \tau^{\prime}\right)=\sum_{\gamma} \int_{0}^{\beta} d \tilde{\tau} \mathbf{A}_{\gamma}^{\alpha}(\tau, \tilde{\tau}) \mathbf{B}_{\beta}^{\gamma}\left(\tilde{\tau}, \tau^{\prime}\right) .
$$

As a consequence, a scalar $R=\mathbf{V}^{\dagger} \mathbf{M F}$, given by the contraction of two first-order tensors $\mathbf{V}^{\alpha}(\tau)$ and $\mathbf{F}^{\alpha}(\tau)$ with a second-order tensor $\mathbf{M}_{\beta}^{\alpha}\left(\tau, \tau^{\prime}\right)$, can be expressed as

$$
R=\sum_{\alpha \beta} \int_{0}^{\beta} \int_{0}^{\beta} d \tau d \tau^{\prime} \mathbf{V}_{\alpha}^{\dagger}(\tau) \mathbf{M}_{\beta}^{\alpha}\left(\tau, \tau^{\prime}\right) \mathbf{F}^{\beta}\left(\tau^{\prime}\right) .
$$

Also, time and space translation invariances imply $\mathbf{M}_{\beta}^{\alpha}(\tau-$ $\left.\tau^{\prime}\right)=\mathbf{M}_{\mathbf{r}_{j}, \nu}^{\mathbf{r}_{i}, \eta}\left(\tau-\tau^{\prime}\right)=\mathbf{M}_{v}^{\eta}\left(\mathbf{r}_{i}-\mathbf{r}_{j}, \tau-\tau^{\prime}\right)$. The connection between the imaginary time domain and the Matsubara frequency space (with $\omega_{n}=\frac{2 \pi}{\beta} n$ ) is established through the Fourier relations

$$
\begin{gathered}
\mathbf{M}_{\beta}^{\alpha}\left(i \omega_{n}\right)=\int_{0}^{\beta} d \tau e^{i \omega_{n} \tau} \mathbf{M}_{\beta}^{\alpha}(\tau), \\
\mathbf{M}_{\beta}^{\alpha}(\tau)=\frac{1}{\beta} \sum_{n=-\infty}^{\infty} e^{-i \omega_{n} \tau} \mathbf{M}_{\beta}^{\alpha}\left(i \omega_{n}\right) .
\end{gathered}
$$

Analogously, real and momentum spaces are related by

$$
\begin{aligned}
\mathbf{M}_{v}^{\eta}(\mathbf{k}, \tau) & =\sum_{\mathbf{r}} e^{-i \mathbf{k} \cdot \mathbf{r}} \mathbf{M}_{v}^{\eta}(\mathbf{r}, \tau), \\
\mathbf{M}_{v}^{\eta}(\mathbf{r}, \tau) & =\frac{1}{N_{s}} \sum_{\mathbf{k}} e^{i \mathbf{k} \cdot \mathbf{r}} \mathbf{M}_{v}^{\eta}(\mathbf{k}, \tau) .
\end{aligned}
$$

Similar conclusions also hold for first-order tensors. Combining space and time translation invariances, in momentum and Matsubara frequency space the products exhibited by Eqs. (A1)-(A3) simplify to

$$
\begin{gathered}
\mathbf{V}^{\eta}=\sum_{\nu} \mathbf{M}_{\nu}^{\eta}\left(\mathbf{k}=\mathbf{0}, i \omega_{0}\right) \mathbf{F}^{v}, \\
\mathbf{M}_{\nu}^{\eta}\left(\mathbf{k}, i \omega_{n}\right)=\sum_{\mu} \mathbf{A}_{\mu}^{\eta}\left(\mathbf{k}, i \omega_{n}\right) \mathbf{B}_{v}^{\mu}\left(\mathbf{k}, i \omega_{n}\right), \\
R=\beta N_{s} \sum_{\eta v} \mathbf{V}_{\eta}^{\dagger} \mathbf{M}_{v}^{\eta}\left(\mathbf{k}=\mathbf{0}, i \omega_{0}\right) \mathbf{F}^{v} .
\end{gathered}
$$

\section{APPENDIX B: TRACES}

\section{General definitions}

The traces presented in the functional formulations are defined as the complete contraction over superindices and a double integration in imaginary time according to

$$
\operatorname{Tr}[\mathbf{M}]=\sum_{\gamma} \int_{0}^{\beta} \int_{0}^{\beta} d \tau d \tau^{\prime} \delta_{\gamma}\left(\tau-\tau^{\prime}\right) \mathbf{M}_{\gamma}^{\gamma}\left(\tau, \tau^{\prime}\right),
$$


where $\delta_{\gamma}(\tau)=\delta_{\mathbf{r}_{i}, v}(\tau)=\delta\left(\tau-(-1)^{v} 0^{-}\right)$with $\quad v=0,1$. The delta function is introduced in order to impose normal ordering of the diagonal Nambu components of M. Considering time and space translation invariances, the trace defined by Eq. (B1) becomes

$$
\begin{aligned}
\operatorname{Tr}[\mathbf{M}] & =\beta N_{s} \sum_{v} \mathbf{M}_{v}^{v}\left[\mathbf{r}_{i}=\mathbf{0}, \tau=(-1)^{v} 0^{-}\right] \\
& =\sum_{v \mathbf{k} n} e^{i \omega_{n}(-1)^{v} 0^{+}} \mathbf{M}_{v}^{v}\left(\mathbf{k}, i \omega_{n}\right) .
\end{aligned}
$$

The summation over Matsubara frequencies within the described limit can impose difficulties depending on the asymptotic behavior of $\mathbf{M}$. In order to improve the convergence properties of such sums, we analyze explicitly its high-frequency expansion in the following.

\section{High-frequency expansion}

We define the high-frequency expansion of

$$
\mathbf{M}\left(\mathbf{k}, i \omega_{n}\right)=\mathcal{M}\left(\mathbf{k}, i \omega_{n}\right)+O\left(\left(i \omega_{n}\right)^{-\left(N_{h}+1\right)}\right)
$$

up to order $N_{h}$ according to

$$
\mathcal{M}\left(\mathbf{k}, i \omega_{n}\right)=\sum_{p=1}^{N_{h}} \mathbf{m}_{p}(\mathbf{k}) Q_{p}\left(i \omega_{n}\right)
$$

where

$$
Q_{p}\left(i \omega_{n}\right)= \begin{cases}\frac{1}{\left(i \omega_{n}\right)^{p}}, & \omega_{n} \neq 0, \\ 0, & \omega_{n}=0 .\end{cases}
$$

By adding and subtracting the term $\operatorname{Tr}[\mathcal{M}]$ in Eq. (B2), the corresponding trace can be expressed as

$$
\begin{aligned}
\operatorname{Tr}[\mathbf{M}]= & \sum_{v \mathbf{k}}\left(\sum_{n}\left[\mathbf{M}\left(\mathbf{k}, i \omega_{n}\right)-\mathcal{M}\left(\mathbf{k}, i \omega_{n}\right)\right]_{v}^{v}\right. \\
& \left.+\beta \mathcal{M}_{v}^{v}\left[\mathbf{k}, \tau=(-1)^{v} 0^{-}\right]\right)
\end{aligned}
$$

since the asymptotic behavior $[\mathbf{M}-\mathcal{M}]_{v}^{v} \sim\left(i \omega_{n}\right)^{-\left(N_{h}+1\right)}$ allows us to drop the exponent present in the trace definition. Considering a finite number $N_{\omega}$ of Matsubara frequencies and a high-frequency expansion of order $N_{h}$, the trace approximation reads as

$$
\begin{aligned}
\operatorname{Tr}[\mathbf{M}] \approx & \sum_{\nu \mathbf{k}}\left(\mathbf{M}_{v}^{v}\left(\mathbf{k}, i \omega_{0}\right)\right. \\
& +\sum_{n=-N_{\omega}}^{N_{\omega}}\left[\mathbf{M}\left(\mathbf{k}, i \omega_{n}\right)-\sum_{p=1}^{N_{h}} \frac{\mathbf{m}_{p}(\mathbf{k})}{\left(i \omega_{n}\right)^{p}}\right]_{v}^{v} \\
& \left.+\beta \sum_{p=1}^{N_{h}}\left[\mathbf{m}_{p}(\mathbf{k})\right]_{\nu}^{v} Q_{p}\left[\mathbf{k}, \tau=(-1)^{v} 0^{-}\right]\right),
\end{aligned}
$$

where the primed sum excludes $\omega_{n}=0$. The calculations presented in this paper employ $N_{\omega}=1000$ with a secondorder $N_{h}=2$ tail expansion.

In order to compute the trace approximation of Eq. (B7), the functions $Q_{p}(\tau)$, Fourier transforms of $Q_{p}\left(i \omega_{n}\right)$, need to be determined. The idea is to visualize them as sums of the residues [46] of a given complex function as follows:

$$
Q_{p}(\tau)=\frac{1}{\beta} \sum_{n=-\infty}^{\infty} \frac{e^{-i \omega_{n} \tau}}{\left(i \omega_{n}\right)^{p}}=\sum_{n=-\infty}^{\infty} \operatorname{Res}\left[\frac{e^{-\tau z}}{z^{p}} h(z), i \omega_{n}\right],
$$

with $h(z)=\left(1-e^{-\beta z}\right)^{-1}$. The poles $z=i \omega_{n}$ are located along the imaginary axis; then, the residue theorem allows us to relate the result to a contour integral, comprehending the imaginary axis of the complex plane

$$
\sum_{n=-\infty}^{\infty} \operatorname{Res}\left[\frac{e^{-\tau z}}{z^{p}} h(z), i \omega_{n}\right]=\oint_{C} \frac{d z}{2 \pi i} \frac{e^{-\tau z}}{z^{p}} h(z) .
$$

By deforming the original contour $C$ into two semicircles of infinite radius this same integral becomes

$$
\oint_{C^{\prime}} \frac{d z}{2 \pi i} \frac{e^{-\tau z}}{z^{p}} h(z)=0,
$$

once this new path $C^{\prime}$ encloses regions free of poles. By inserting Eq. (B10) into Eq. (B9) and comparing to Eq. (B8), $Q_{p}(\tau)$ is determined simply by $n=0$

$$
Q_{p}(\tau)=-\operatorname{Res}\left[\frac{e^{-\tau z}}{z^{p}} h(z), 0\right] .
$$

The relevant pole $z=0$ is of order $p+1$; hence the corresponding residue is given by the formula

$$
Q_{p}(\tau)=-\frac{1}{p !} \lim _{z \rightarrow 0}\left(\frac{d}{d z}\right)^{p} z e^{-\tau z} h(z),
$$

with the following first-order terms:

$$
\begin{gathered}
Q_{1}(\tau)=(2 \tau-\beta) /(2 \beta), \\
Q_{2}(\tau)=\left(-6 \tau^{2}+6 \beta \tau-\beta^{2}\right) /(12 \beta) .
\end{gathered}
$$

Since the functions $Q_{p}(\tau)$ are periodic on the interval $\tau \in$ $(0, \beta)$, the respective zero time limits are

$$
\begin{gathered}
Q_{1}\left[\tau=(-1)^{v} 0^{-}\right]=(-1)^{v} / 2, \\
Q_{2}\left[\tau=(-1)^{v} 0^{-}\right]=-\beta / 12 .
\end{gathered}
$$

\section{Trace of logarithm}

Besides the already mentioned ordinary traces, the selfenergy functionals also contain traces regarding the logarithm of the Green's function G. Actually, in order to guarantee the correct noninteracting limit, a regularization factor must be included. Therefore, we define the regularized Matsubara trace logarithm functional $\mathcal{L}[\mathbf{G}]$,

$$
\mathcal{L}[\mathbf{G}]=\frac{1}{2} \operatorname{Tr} \ln \left[-\mathbf{G}^{-1}\right]-\frac{1}{2} \operatorname{Tr} \ln \left[-\mathbf{G}_{r}^{-1}\right],
$$

with the regularization carried by the second-order tensor

$$
\mathbf{G}_{r}\left(i \omega_{n}\right)= \begin{cases}-\beta \mathbf{1}, & \omega_{n}=0 \\ \frac{\sigma_{z}}{i \omega_{n}}, & \omega_{n} \neq 0 .\end{cases}
$$

According to Eq. (B2), such trace is represented as

$$
\mathcal{L}[\mathbf{G}]=-\frac{1}{2} \sum_{v \mathbf{k} n} e^{i \omega_{n}(-1)^{v} 0^{+}}\left\{\ln \left[\mathbf{G}_{r}^{-1} \mathbf{G}\left(\mathbf{k}, i \omega_{n}\right)\right]\right\}_{v}^{v},
$$


and the approximation described by Eq. (B7) yields

$$
\begin{aligned}
\mathcal{L}[\mathbf{G}] \approx & -\frac{1}{2} \sum_{\nu \mathbf{k}}\left(\ln \left[-\mathbf{G}\left(\mathbf{k}, i \omega_{0}\right) / \beta\right]_{\nu}^{v}\right. \\
& +\sum_{n=-N_{\omega}}^{N_{\omega}}\left[\ln \left[\left(\sigma_{z} i \omega_{n}\right) \mathbf{G}\left(\mathbf{k}, i \omega_{n}\right)\right]-\sum_{p=1}^{N_{h}} \frac{\mathbf{q}_{p}(\mathbf{k})}{\left(i \omega_{n}\right)^{p}}\right]_{\nu}^{v} \\
& \left.+\beta \sum_{p=1}^{N_{h}}\left[\mathbf{q}_{p}(\mathbf{k})\right]_{\nu}^{v} Q_{p}\left[\mathbf{k}, \tau=(-1)^{v} 0^{-}\right]\right)
\end{aligned}
$$

The terms $\mathbf{q}_{p}(\mathbf{k})$ are the coefficients from the high-frequency expansion of $\ln \left[\left(\sigma_{z} i \omega_{n}\right) \mathbf{G}\left(\mathbf{k}, i \omega_{n}\right)\right]=\sum_{p=1}^{\infty} \frac{\mathbf{q}_{p}(\mathbf{k})}{\left(i \omega_{n}\right)^{p}}$ given explicitly by

$$
\begin{gathered}
\mathbf{q}_{1}(\mathbf{k})=\sigma_{z} \mathbf{c}_{2}(\mathbf{k}), \\
\mathbf{q}_{2}(\mathbf{k})=\sigma_{z} \mathbf{c}_{3}(\mathbf{k})-\frac{\left[\sigma_{z} \mathbf{c}_{2}(\mathbf{k})\right]^{2}}{2},
\end{gathered}
$$

until second order, where $\mathbf{c}_{p}(\mathbf{k})$ are the coefficients in the high-frequency expansion of the Green's function

$$
\mathbf{G}\left(i \omega_{n}\right)=\sum_{p=1}^{\infty} \frac{\mathbf{c}_{p}(\mathbf{k})}{\left(i \omega_{n}\right)^{p}} .
$$

Hence, using second-order tail corrections, the Matsubara trace logarithm exposed in Eq. (B19) reads as

$$
\begin{aligned}
\mathcal{L}[\mathbf{G}] \approx & -\frac{1}{2} \sum_{\nu, \mathbf{k}}\left(\ln \left[-\mathbf{G}\left(\mathbf{k}, i \omega_{0}\right) / \beta\right]+\frac{\beta}{2} \mathbf{c}_{2}(\mathbf{k})-\frac{\beta^{2}}{12} \mathbf{q}_{2}(\mathbf{k})\right. \\
& \left.+\sum_{n=-N_{\omega}}^{N_{\omega}}\left[\ln \left[\left(\sigma_{z} i \omega_{n}\right) \mathbf{G}\left(\mathbf{k}, i \omega_{n}\right)\right]-\frac{\mathbf{q}_{2}(\mathbf{k})}{\left(i \omega_{n}\right)^{2}}\right]\right)_{\nu}^{v} .
\end{aligned}
$$

\section{APPENDIX C: REFERENCE SYSTEM}

In the basis of local occupation number states, we generate matrix representations for the reference system Hamiltonian $H^{\prime}$ of Eq. (24) and the bosonic creation and annihilation operators $b^{\dagger}$ and $b$. A cutoff $N_{\max }=10$ bounding the occupation number from above is introduced. The diagonalization of the Hamiltonian $H^{\prime}$ provides its eigenvalues $E_{n}$ and eigenvectors $|n\rangle$. Given these procedures, the partition function is written as

$$
\mathcal{Z}^{\prime}=\sum_{n} e^{-\beta E_{n}},
$$

from which we derive the reference system free energy

$$
\Omega^{\prime}=-\frac{1}{\beta} \ln \left[\mathcal{Z}^{\prime}\right] .
$$

Regarding the propagators, the static expectation value of $\mathbf{b}$, defined in Eq. (8), is given by

$$
\boldsymbol{\Phi}^{\prime}=\langle\mathbf{b}\rangle=\frac{1}{\mathcal{Z}^{\prime}} \sum_{n} e^{-\beta E_{n}}\langle n|\mathbf{b}| n\rangle,
$$

and the connected Green's function of Eq. (9)

$$
\mathbf{G}^{\prime}(\tau)=-\left\langle\mathbf{b}(\tau) \mathbf{b}^{\dagger}\right\rangle+\boldsymbol{\Phi}^{\prime} \boldsymbol{\Phi}^{\prime \dagger}
$$

expressed in the Matsubara frequency space yields

$$
\begin{aligned}
\mathbf{G}^{\prime}\left(i \omega_{n}\right)= & \frac{1}{\mathcal{Z}^{\prime}} \sum_{n, m} \frac{\left(e^{-\beta E_{n}}-e^{-\beta E_{m}}\right)}{i \omega_{n}+E_{n}-E_{m}}\langle n|\mathbf{b}| m\rangle\left\langle m\left|\mathbf{b}^{\dagger}\right| n\right\rangle \\
& -\delta_{\omega_{n}, 0} \beta \frac{1}{\mathcal{Z}^{\prime}} \sum_{n} e^{-\beta E_{n}}\langle n|\mathbf{b}| n\rangle\left\langle n\left|\mathbf{b}^{\dagger}\right| n\right\rangle \\
& +\delta_{\omega_{n}, 0} \beta \boldsymbol{\Phi}^{\prime} \boldsymbol{\Phi}^{\prime \dagger}
\end{aligned}
$$

The noninteracting Green's function is determined by setting $U=0$ in Eq. (24),

$$
\mathbf{G}_{0}^{\prime-1}\left(i \omega_{n}\right)=\sigma_{z} i \omega_{n}+\mathbf{1} \mu-\mathbf{\Delta},
$$

while the self-energies follow from the Dyson equations, Eqs. (19) and (20), as

$$
\begin{gathered}
\boldsymbol{\Sigma}_{1 / 2}^{\prime}=\mathbf{F}^{\prime}-\mathbf{G}_{0}^{\prime-1}\left(i \omega_{0}\right) \boldsymbol{\Phi}^{\prime} \\
\boldsymbol{\Sigma}^{\prime}\left(i \omega_{n}\right)=\mathbf{G}_{0}^{\prime-1}\left(i \omega_{n}\right)-\mathbf{G}^{\prime-1}\left(i \omega_{n}\right) .
\end{gathered}
$$

\section{APPENDIX D: LATTICE SYSTEM}

Considering the lattice system, the noninteracting Green's function satisfies

$$
\mathbf{G}_{0}^{-1}\left(\mathbf{k}, i \omega_{n}\right)=\sigma_{z} i \omega_{n}+\mathbf{1}\left(\mu-\epsilon_{\mathbf{k}}\right),
$$

where $\epsilon_{\mathbf{k}}$ is the energy dispersion relation

$$
\epsilon_{\mathbf{k}}=-2 J \sum_{i=1}^{d} \cos \left(k_{i} a\right)
$$

for a hypercubic lattice in $d$ dimensions. Also, the interacting lattice Green's function follows from Eq. (20) as

$$
\mathbf{G}^{-1}\left(\mathbf{k}, i \omega_{n}\right)=\mathbf{G}_{0}^{-1}\left(\mathbf{k}, i \omega_{n}\right)-\boldsymbol{\Sigma}^{\prime}\left(i \omega_{n}\right)
$$

evaluated at the reference system self-energy $\boldsymbol{\Sigma}^{\prime}$. According to Eq. (19), the other Dyson equation implies

$$
\boldsymbol{\Phi}=-\mathbf{G}_{\mathbf{0}}\left(i \omega_{0}\right) \boldsymbol{\Sigma}_{1 / 2}^{\prime},
$$

taking into account that $\mathbf{F}=\mathbf{0}$, since there is no symmetry breaking field on the complete lattice.

\section{APPENDIX E: HIGH-FREQUENCY EXPANSION OF THE GREEN'S FUNCTION}

To complete the evaluation of the trace log functional of Eq. (B23), the coefficients $\mathbf{c}_{p}$, regarding the tail expansion of the Green's function in Eq. (B22), remain to be determined for both reference and lattice systems.

\section{Reference system}

By expressing the Matsubara Green's function as a Fourier transform of the imaginary time Green's function, successive 
integration by parts yields

$$
\begin{aligned}
\mathbf{G}^{\prime}\left(i \omega_{n}\right) & =\int_{0}^{\beta} d \tau e^{i \omega_{n} \tau} \mathbf{G}^{\prime}(\tau) \\
& =\sum_{p=0}^{\infty}(-1)^{p} \frac{\partial_{\tau}^{p} \mathbf{G}^{\prime}\left(\beta^{-}\right)-\partial_{\tau}^{p} \mathbf{G}^{\prime}\left(0^{+}\right)}{\left(i \omega_{n}\right)^{p+1}} .
\end{aligned}
$$

Therefore, the high-frequency expansion coefficients take the form

$$
\mathbf{c}_{p+1}^{\prime}=(-1)^{p}\left[\partial_{\tau}^{p} \mathbf{G}^{\prime}\left(\beta^{-}\right)-\partial_{\tau}^{p} \mathbf{G}^{\prime}\left(0^{+}\right)\right] .
$$

From the definition of the Green's function as the timeordered expectation value of Eq. (C4) and the equation of motion satisfied by $\mathbf{b}(\tau)$

$$
\partial_{\tau} \mathbf{b}(\tau)=\left[H^{\prime}, \mathbf{b}(\tau)\right],
$$

we extract the imaginary time derivative of $\mathbf{G}^{\prime}(\tau)$ :

$$
\partial_{\tau} \mathbf{G}(\tau)=-\left\langle\left[H^{\prime}, \mathbf{b}(\tau)\right] \mathbf{b}^{\dagger}\right\rangle .
$$

By induction on Eq. (E3) and Eq. (E4), the derivative of order $p$ reads as

$$
\partial_{\tau}^{p} \mathbf{G}(\tau)=-\left\langle\left[\left[H^{\prime}, \mathbf{b}(\tau)\right]\right]^{(p)} \mathbf{b}^{\dagger}\right\rangle,
$$

with $\left[\left[H^{\prime}, \mathbf{b}(\tau)\right]\right]^{(p)}=[H, \ldots,[H,[H, \mathbf{b}(\tau)]] \ldots]$ the left side commutator of $H^{\prime}$ with $\mathbf{b}(\tau)$ applied $p$ times. The evaluation of such derivatives at the imaginary times $\tau=0^{+}$and $\tau=\beta^{-}$allows us to fix the time ordering

$$
\begin{aligned}
\partial_{\tau}^{p} \mathbf{G}^{\prime}\left(0^{+}\right) & =-\left\langle\left[\left[H^{\prime}, \mathbf{b}\right]\right]^{(p)} \mathbf{b}^{\dagger}\right\rangle, \\
\partial_{\tau}^{p} \mathbf{G}^{\prime}\left(\beta^{-}\right) & =-\left\langle\mathbf{b}^{\dagger}\left[\left[H^{\prime}, \mathbf{b}\right]\right]^{(p)}\right\rangle .
\end{aligned}
$$

Inserting these results into Eq. (E2), the desired coefficients simplify to

$$
\mathbf{c}_{p+1}^{\prime}=(-1)^{p}\left\langle\left[\left[\left[H^{\prime}, \mathbf{b}\right]\right]^{(p)}, \mathbf{b}^{\dagger}\right]\right\rangle .
$$

Explicitly, the first-order terms are

$$
\begin{gathered}
\mathbf{c}_{1}^{\prime}=\left\langle\left[\mathbf{b}, \mathbf{b}^{\dagger}\right]\right\rangle=\sigma_{z}, \\
\mathbf{c}_{2}^{\prime}=-\left\langle\left[\left[H^{\prime}, \mathbf{b}\right], \mathbf{b}^{\dagger}\right]\right\rangle, \\
\mathbf{c}_{3}^{\prime}=\left\langle\left[\left[H^{\prime},\left[H^{\prime}, \mathbf{b}\right]\right], \mathbf{b}^{\dagger}\right]\right\rangle .
\end{gathered}
$$

\section{Lattice system}

According to Eqs. (D1) and (D3), the lattice Green's function $\mathbf{G}\left(\mathbf{k}, i \omega_{n}\right)$ presents a tail expansion of the form

$$
\begin{aligned}
& \mathbf{G}\left(\mathbf{k}, i \omega_{n}\right)=\frac{\sigma_{z}}{i \omega_{n}}+\frac{\sigma_{z}\left[\mathbf{1}\left(\epsilon_{\mathbf{k}}-\mu\right)+\mathbf{s}_{0}^{\prime}\right] \sigma_{z}}{\left(i \omega_{n}\right)^{2}} \\
& +\frac{\sigma_{z} \mathbf{s}_{1}^{\prime} \sigma_{z}+\left\{\sigma_{z}\left[\mathbf{1}\left(\epsilon_{\mathbf{k}}-\mu\right)+\mathbf{s}_{0}^{\prime}\right]\right\}^{2} \sigma_{z}}{\left(i \omega_{n}\right)^{3}}+O\left(\frac{1}{\left(i \omega_{n}\right)^{4}}\right),
\end{aligned}
$$

where $\mathbf{s}_{p}^{\prime}$ are the high-frequency expansion coefficients of the reference system's self-energy $\boldsymbol{\Sigma}^{\prime}\left(i \omega_{n}\right)=\sum_{p=1}^{\infty} \frac{\mathbf{s}_{p}^{\prime}}{\left(i \omega_{n}\right)^{p}}$. Hence the first-order terms are

$$
\begin{gathered}
\mathbf{c}_{1}(\mathbf{k})=\sigma_{z}, \\
\mathbf{c}_{2}(\mathbf{k})=\sigma_{z}\left[\mathbf{1}\left(\epsilon_{\mathbf{k}}-\mu\right)+\mathbf{s}_{0}^{\prime}\right] \sigma_{z}, \\
\mathbf{c}_{3}(\mathbf{k})=\sigma_{z} \mathbf{s}_{1}^{\prime} \sigma_{z}+\left\{\sigma_{z}\left[\mathbf{1}\left(\epsilon_{\mathbf{k}}-\mu\right)+\mathbf{s}_{0}^{\prime}\right]\right\}^{2} \sigma_{z} .
\end{gathered}
$$

To determine the coefficients $\mathbf{s}_{p}^{\prime}$ of the self-energy $\boldsymbol{\Sigma}^{\prime}\left(i \omega_{n}\right)=$ $\mathbf{G}_{0}^{\prime-1}\left(i \omega_{n}\right)-\mathbf{G}^{\prime-1}\left(i \omega_{n}\right)$, we need to compute the inverse of $\mathbf{G}^{\prime}$, given by

$$
\begin{aligned}
\mathbf{G}^{\prime-1}\left(i \omega_{n}\right)= & {\left[\frac{\sigma_{z}}{i \omega_{n}}+\sum_{p=2}^{\infty} \frac{\mathbf{c}_{p}^{\prime}}{\left(i \omega_{n}\right)^{p}}\right]^{-1} } \\
= & \sigma_{z} i \omega_{n}-\sigma_{z} \mathbf{c}_{2}^{\prime} \sigma_{z} \\
& +\frac{-\sigma_{z} \mathbf{c}_{3}^{\prime} \sigma_{z}+\left(\sigma_{z} \mathbf{c}_{2}^{\prime}\right)^{2} \sigma_{z}}{i \omega_{n}}+O\left(\frac{1}{\left(i \omega_{n}\right)^{2}}\right) .
\end{aligned}
$$

Then, the previous result combined to Eq. (C6) implies

$$
\begin{aligned}
\boldsymbol{\Sigma}^{\prime-1}\left(i \omega_{n}\right)= & \mathbf{G}_{0}^{\prime-1}\left(i \omega_{n}\right)-\mathbf{G}^{\prime-1}\left(i \omega_{n}\right) \\
= & \mathbf{1} \mu-\boldsymbol{\Delta}+\sigma_{z} \mathbf{c}_{2}^{\prime} \sigma_{z} \\
& -\frac{-\sigma_{z} \mathbf{c}_{3}^{\prime} \sigma_{z}+\left(\sigma_{z} \mathbf{c}_{2}^{\prime}\right)^{2} \sigma_{z}}{i \omega_{n}}+O\left(\frac{1}{\left(i \omega_{n}\right)^{2}}\right) .
\end{aligned}
$$

Consequently, the first-order coefficients of the self-energy tail expansion can be written as

$$
\begin{gathered}
\mathbf{s}_{0}^{\prime}=\mathbf{1} \mu-\boldsymbol{\Delta}+\sigma_{z} \mathbf{c}_{2}^{\prime} \sigma_{z}, \\
\mathbf{s}_{1}^{\prime}=\sigma_{z} \mathbf{c}_{3}^{\prime} \sigma_{z}-\left(\sigma_{z} \mathbf{c}_{2}^{\prime}\right)^{2} \sigma_{z} .
\end{gathered}
$$

From these terms, the desired lattice Green's function coefficients are evaluated according to Eqs. (E12)-(E14).

\section{APPENDIX F: EQUATION OF STATE AND PRESSURE}

Considering the two-dimensional setup discussed in Sec. VI, the total pressure $P_{\text {total }}$ is evaluated by integrating the Gibbs-Duhem relation $d P=\rho d \mu+s d T$ at fixed temperature $T$ over the lattice

$$
P_{\text {total }}=\int_{-\infty}^{\mu_{0}} \rho d \mu=-\int_{0}^{\infty} \rho(r) \frac{d \mu}{d r} d r .
$$

For an isotropic harmonic trap in the $x y$ plane, $\mu=\mu_{0}-$ $\frac{1}{2} m \omega^{2} r^{2}$ and $\frac{d \mu}{d r}=-m \omega^{2} r$; then Eq. (F1) becomes

$$
P_{\text {total }}=m \omega^{2} \int_{0}^{\infty} \rho(r) r d r .
$$

The total number of particles $N_{\text {total }}$ is obtained by integration of the local density $\rho(r)$ according to

$$
N_{\text {total }}=2 \pi \int_{0}^{\infty} \rho(r) r d r .
$$

Comparing both Eqs. (F2) and (F3), we deduce the equation of state,

$$
P_{\text {total }}=\frac{m \omega^{2}}{2 \pi} N_{\text {total }},
$$

valid for $2 \mathrm{D}$ gases including an isotropic harmonic confinement $[65,66]$. There is also another interesting observation concerning the value of the fixed total pressure. Since the density $\rho$ is a derivative of the free energy $\Omega$, see Eq. (25), 
we can write $P_{\text {total }}$ as

$$
\begin{aligned}
P_{\text {total }} & =-\int_{-\infty}^{\mu_{0}} \frac{1}{V}\left(\frac{\partial \Omega}{\partial \mu}\right)_{T} d \mu \\
& =\frac{1}{V}\left[\Omega(\mu=-\infty)-\Omega\left(\mu_{0}\right)\right] .
\end{aligned}
$$

Considering that $\Omega(\mu=-\infty)=0$ and $-P V=\Omega$, the total pressure is equal to the local pressure at the center of the trap

$$
P_{\text {total }}=P\left(\mu_{0}\right)=P(r=0),
$$

as Fig. 5(a) confirms.
[1] M. H. Anderson, J. R. Ensher, M. R. Matthews, C. E. Wieman, and E. A. Cornell, Science 269, 198 (1995).

[2] K. B. Davis, M. O. Mewes, M. R. Andrews, N. J. van Druten, D. S. Durfee, D. M. Kurn, and W. Ketterle, Phys. Rev. Lett. 75, 3969 (1995).

[3] O. Dutta, M. Gajda, P. Hauke, M. Lewenstein, D.-S. Lühmann, B. A. Malomed, T. Sowiński, and J. Zakrzewski, Rep. Prog. Phys. 78, 066001 (2015).

[4] M. Lewenstein, A. Sanpera, V. Ahufinger, B. Damski, A. Sen(De), and U. Sen, Adv. Phys. 56, 243 (2007).

[5] D. Jaksch, H.-J. Briegel, J. I. Cirac, C. W. Gardiner, and P. Zoller, Phys. Rev. Lett. 82, 1975 (1999).

[6] J. I. Cirac and P. Zoller, Phys. Today 57(3), 38 (2004).

[7] M. Greiner, O. Mandel, T. Esslinger, T. W. Hänsch, and I. Bloch, Nature (London) 415, 39 (2002).

[8] I. Bloch, Nat. Phys. 1, 23 (2005).

[9] D. Jaksch and P. Zoller, Ann. Phys. (NY) 315, 52 (2005).

[10] W. Hofstetter and T. Qin, J. Phys. B: At., Mol., Opt. Phys. 51, 082001 (2018).

[11] E. J. Mueller, Phys. Rev. A 70, 041603(R) (2004).

[12] E. Kapit and E. Mueller, Phys. Rev. A 83, 033625 (2011).

[13] P. Windpassinger and K. Sengstock, Rep. Prog. Phys. 76, 086401 (2013).

[14] M. P. A. Fisher, P. B. Weichman, G. Grinstein, and D. S. Fisher, Phys. Rev. B 40, 546 (1989).

[15] D. Jaksch, C. Bruder, J. I. Cirac, C. W. Gardiner, and P. Zoller, Phys. Rev. Lett. 81, 3108 (1998).

[16] S. Sachdev, Quantum Phase Transitions (Cambridge University Press, Cambridge, UK, 2011).

[17] G. S. Kell, J. Chem. Eng. Data 12, 66 (1967).

[18] P. G. Debenedetti, J. Phys.: Condens. Matter 15, R1669(R) (2003).

[19] R. J. Donnelly and C. F. Barenghi, J. Phys. Chem. Ref. Data 27, 1217 (1998).

[20] F. Caupin, S. Balibar, and H. J. Maris, Phys. Rev. Lett. 87, 145302 (2001).

[21] P. H. Poole, F. Sciortino, U. Essmann, and H. E. Stanley, Nature (London) 360, 324 (1992).

[22] P. Gallo, K. Amann-Winkel, C. A. Angell, M. A. Anisimov, F. Caupin, C. Chakravarty, E. Lascaris, T. Loerting, A. Z. Panagiotopoulos, J. Russo, J. A. Sellberg, H. E. Stanley, H. Tanaka, C. Vega, L. Xu, and L. G. M. Pettersson, Chem. Rev. 116, 7463 (2016).

[23] H. E. Stanley, P. Kumar, G. Franzese, L. Xu, Z. Yan, M. G. Mazza, S. V. Buldyrev, S.-H. Chen, and F. Mallamace, Eur. Phys. J.: Spec. Top. 161, 1 (2008).

[24] J. N. Glosli and F. H. Ree, Phys. Rev. Lett. 82, 4659 (1999).

[25] S. Sastry and C. Austen Angell, Nat. Mater. 2, 739 (2003).

[26] I. Saika-Voivod, F. Sciortino, T. Grande, and P. H. Poole, Philos. Trans. R. Soc., A 363, 525 (2005).
[27] G. Monaco, S. Falconi, W. A. Crichton, and M. Mezouar, Phys. Rev. Lett. 90, 255701 (2003).

[28] R. Kurita and H. Tanaka, J. Phys.: Condens. Matter 17, L293 (2005).

[29] K.-i. Murata and H. Tanaka, Nat. Commun. 4, 2844 (2013).

[30] A. Taschin, P. Bartolini, R. Eramo, R. Righini, and R. Torre, Nat. Commun. 4, 2401 (2013).

[31] D. T. Limmer and D. Chandler, J. Chem. Phys. 138, 214504 (2013).

[32] D. Hügel, P. Werner, L. Pollet, and H. U. R. Strand, Phys. Rev. B 94, 195119 (2016).

[33] L. Pollet, Rep. Prog. Phys. 75, 094501 (2012).

[34] I. Bloch, J. Dalibard, and W. Zwerger, Rev. Mod. Phys. 80, 885 (2008).

[35] W. Zwerger, J. Opt. B: Quantum Semiclass. Opt. 5, S9 (2003).

[36] P. B. Blakie and C. W. Clark, J. Phys. B: At., Mol., Opt. Phys. 37, 1391 (2004).

[37] W. S. Bakr, J. I. Gillen, A. Peng, S. Fölling, and M. Greiner, Nature (London) 462, 74 (2009).

[38] J. F. Sherson, C. Weitenberg, M. Endres, M. Cheneau, I. Bloch, and S. Kuhr, Nature (London) 467, 68 (2010).

[39] M. Potthoff, Eur. Phys. J. B 32, 429 (2003).

[40] K. Byczuk and D. Vollhardt, Phys. Rev. B 77, 235106 (2008).

[41] A. Hubener, M. Snoek, and W. Hofstetter, Phys. Rev. B 80, 245109 (2009).

[42] W.-J. Hu and N.-H. Tong, Phys. Rev. B 80, 245110 (2009).

[43] P. Anders, E. Gull, L. Pollet, M. Troyer, and P. Werner, New J. Phys. 13, 075013 (2011).

[44] E. Arrigoni, M. Knap, and W. von der Linden, Phys. Rev. B 84, 014535 (2011).

[45] D. Hügel, H. U. R. Strand, and L. Pollet, Quantum Sci. Technol. 3, 034006 (2018).

[46] A. L. Fetter and J. D. Walecka, Quantum Theory of ManyParticle Systems (Dover Publications, Mineola, NY, 1971), p. 250 .

[47] J. Negele and H. Orland, Quantum Many-Particle Systems (Perseus Books, New York, 1998), p. 237.

[48] J. Zinn-Justin, Path Integrals in Quantum Mechanics (Oxford University Press, Oxford, 2004).

[49] G. Baym and L. P. Kadanoff, Phys. Rev. 124, 287 (1961).

[50] G. Baym, Phys. Rev. 127, 1391 (1962).

[51] C. De Dominicis and P. C. Martin, J. Math. Phys. 5, 14 (1964).

[52] C. De Dominicis and P. C. Martin, J. Math. Phys. 5, 31 (1964).

[53] J. M. Luttinger and J. C. Ward, Phys. Rev. 118, 1417 (1960).

[54] G. Stefanucci and R. van Leeuwen, Nonequilibrium ManyBody Theory of Quantum Systems (Cambridge University Press, Cambridge, UK, 2013), p. 249. 
[55] J. Berges, S. Borsányi, U. Reinosa, and J. Serreau, Ann. Phys. (NY) 320, 344 (2005).

[56] J. F. Rentrop, V. Meden, and S. G. Jakobs, Phys. Rev. B 93, 195160 (2016).

[57] See Supplemental Material at http://link.aps.org/supplemental/ 10.1103/PhysRevA.102.033331 for the numerical code regarding the implementation of the self-energy functional theory.

[58] N. Gemelke, X. Zhang, C. L. Hung, and C. Chin, Nature (London) 460, 995 (2009).

[59] K. Xu, Y. Liu, J. R. Abo-Shaeer, T. Mukaiyama, J. K. Chin, D. E. Miller, W. Ketterle, K. M. Jones, and E. Tiesinga, Phys. Rev. A 72, 043604 (2005).
[60] C. Chin, R. Grimm, P. Julienne, and E. Tiesinga, Rev. Mod. Phys. 82, 1225 (2010).

[61] X. Zhang, C.-L. Hung, S.-K. Tung, and C. Chin, Science 335, 1070 (2012).

[62] E. O. Rizzatti, M. S. Gomes Filho, M. Malard, and M. A. A. Barbosa, Physica A 518, 323 (2019).

[63] I. Bloch, Nat. Phys. 14, 1159 (2018).

[64] T. Gericke, P. Würtz, D. Reitz, T. Langen, and H. Ott, Nat. Phys. 4, 949 (2008).

[65] S. Nascimbène, N. Navon, F. Chevy, and C. Salomon, New J. Phys. 12, 103026 (2010).

[66] T.-L. Ho and Q. Zhou, Nat. Phys. 6, 131 (2010). 